\title{
SCHATTEN $p$-CLASS PROPERTY OF PSEUDODIFFERENTIAL OPERATORS WITH SYMBOLS IN MODULATION SPACES
}

\author{
MASAHARU KOBAYASHI AND AKIHIKO MIYACHI
}

\begin{abstract}
It is proved that the pseudodifferential operators $\sigma_{t}(X, D)$ belong to the Schatten $p$-class $C_{p}, 0<p \leq 2$, if the symbol $\sigma(x, \omega)$ is in certain modulation spaces on $\mathbf{R}_{x}^{d} \times \mathbf{R}_{\omega}^{d}$.
\end{abstract}

\section{$\S 1$. Introduction}

The Schatten $p$-class $C_{p}$ is the class of compact operators introduced by von Neumann and Schatten, which is defined as follows (see [2], [20]). Let $0<p<\infty$, and let $A$ be a compact operator on $L^{2}\left(\mathbf{R}^{d}\right)$. If the singular values $s_{j}(A)$ of $A$, that is, the eigenvalues of the positive compact operator $|A|=\left(A^{*} A\right)^{1 / 2}$, satisfy $\|A\|_{C_{p}}=\left(\sum_{j=1}^{\infty} s_{j}(A)^{p}\right)^{1 / p}<\infty$, then $A$ is said to be in the Schatten $p$-class $C_{p}$, and we denote $A \in C_{p}$.

If $\sigma(x, \omega)$ is a function on $\mathbf{R}_{x}^{d} \times \mathbf{R}_{\omega}^{d}$ and $t \in \mathbf{R}$, then the pseudodifferential operator $\sigma_{t}(X, D)$ is defined by

$$
\begin{aligned}
\sigma_{t}(X, D) f(x) & =\iint_{\mathbf{R}^{2 d}} \sigma((1-t) x+t y, \omega) e^{2 \pi i(x-y) \omega} f(y) d y d \omega \\
& =\iint_{\mathbf{R}^{2 d}} \widehat{\sigma}(\xi, \eta) e^{2 \pi i(x+t \eta) \xi} f(\eta+x) d \xi d \eta
\end{aligned}
$$

The operator $\sigma_{t}(X, D)$ is a generalization of the Kohn-Nirenberg correspondence $(t=0)$

$$
\sigma(X, D) f(x)=\int_{\mathbf{R}^{d}} \sigma(x, \omega) \widehat{f}(\omega) e^{2 \pi i x \omega} d \omega,
$$

and the Weyl correspondence $(t=1 / 2)$

$$
\sigma^{W}(X, D) f(x)=\iint_{\mathbf{R}^{2 d}} \sigma\left(\frac{x+y}{2}, \omega\right) e^{2 \pi i(x-y) \omega} f(y) d y d \omega .
$$

Received November 8, 2010. Accepted August 23, 2011.

2010 Mathematics Subject Classification. 42B35, 47B10.

(C) 2012 by The Editorial Board of the Nagoya Mathematical Journal 
We will consider sufficient conditions on symbols $\sigma(x, \omega)$ to ensure that the operator $\sigma_{t}(X, D)$ belongs to the Schatten $p$-class $C_{p}$. These types of issues have been studied by a number of authors using a large variety of methods (see [19], [18], [1], and [9], [10], [12] cited below). In this article, we consider the pseudodifferential operators with symbols in the modulation spaces, which are defined as follows.

Definition 1.1 [3, Definition 6.1]. Fix a nonzero $\varphi \in \mathcal{S}\left(\mathbf{R}^{n}\right), 0<p, q \leq$ $\infty$, and a positive function $m$ on $\mathbf{R}^{n} \times \mathbf{R}^{n}$ which satisfies

$$
m\left(z+z^{\prime}, \zeta+\zeta^{\prime}\right) \leq C m(z, \zeta)\left(1+\left|z^{\prime}\right|+\left|\zeta^{\prime}\right|\right)^{s}, \quad z, \zeta, z^{\prime}, \zeta^{\prime} \in \mathbf{R}^{n}
$$

for some constants $C>0$ and $s \geq 0$. Then the modulation space $M_{m}^{p, q}\left(\mathbf{R}^{n}\right)$ consists of all tempered distributions $f \in \mathcal{S}^{\prime}\left(\mathbf{R}^{n}\right)$ such that the quasi-norm

$$
\|f\|_{M_{m}^{p, q}}=\left(\int_{\mathbf{R}^{n}}\left(\int_{\mathbf{R}^{n}}\left|f *\left(M_{\zeta} \varphi\right)(z)\right|^{p} m(z, \zeta)^{p} d z\right)^{q / p} d \zeta\right)^{1 / q}
$$

is finite, with obvious modifications if $p$ or $q=\infty$.

It is known that $M_{m}^{p, q}\left(\mathbf{R}^{n}\right)$ and the equivalence class of the quasi-norm do not depend on the choice of the function $\varphi$ (see [3], [5], [7]). We simply write $M^{p, q}\left(\mathbf{R}^{n}\right)$ instead of $M_{m}^{p, q}\left(\mathbf{R}^{n}\right)$ when $m \equiv 1$.

In the present paper, we will use the modulation space on $\mathbf{R}^{2 d}$. We will frequently write the elements of $\mathbf{R}^{2 d}$ as

$$
z=\left(z_{1}, z_{2}\right), \quad z_{1}, z_{2} \in \mathbf{R}^{d}
$$

Concerning the modulation spaces and the Schatten class, the following theorem is known.

Theorem A (see [9], [12]). Let $0<p<2$. Then the pseudodifferential operators $\sigma_{0}(X, D)=\sigma(X, D)$ and $\sigma_{1 / 2}(X, D)=\sigma^{W}(X, D)$ are in the class $C_{p}$ if $\sigma \in M_{m}^{2,2}\left(\mathbf{R}^{2 d}\right)$ with

$$
m(z, \zeta)=\left(1+\left|z_{1}\right|+\left|z_{2}\right|+\left|\zeta_{1}\right|+\left|\zeta_{2}\right|\right)^{s}
$$

and $s>(2 d / p)-d$.

As for the modulation space $M_{m}^{2,2}\left(\mathbf{R}^{2 d}\right)$ of this theorem, the following identification is known (see [7, Proposition 11.3.1]). If $m$ is defined by (1.2), 
with $s \geq 0$ in general, then

$$
\begin{aligned}
\|\sigma\|_{M_{m}^{2,2}} \approx & \left\|\left(1+\left|z_{1}\right|+\left|z_{2}\right|\right)^{s} \sigma\left(z_{1}, z_{2}\right)\right\|_{L_{z_{1}, z_{2}}^{2}} \\
& +\left\|\left(1+\left|\zeta_{1}\right|+\left|\zeta_{2}\right|\right)^{s} \widehat{\sigma}\left(\zeta_{1}, \zeta_{2}\right)\right\|_{L_{\zeta_{1}, \zeta_{2}}^{2}},
\end{aligned}
$$

where $\widehat{\sigma}$ denotes the Fourier transform defined by

$$
\widehat{\sigma}\left(\zeta_{1}, \zeta_{2}\right)=\int_{\mathbf{R}^{d} \times \mathbf{R}^{d}} \sigma\left(z_{1}, z_{2}\right) e^{-2 \pi i\left(z_{1} \zeta_{1}+z_{2} \zeta_{2}\right)} d z_{1} d z_{2} .
$$

One of the main purposes of the present paper is to give a refinement of Theorem A, which reads as follows.

TheOREM 1.2. Let $0<p<2$, let $s>(2 d / p)-d$, and let $t \in \mathbf{R}$. If $\sigma \in$ $M_{m}^{2,2}\left(\mathbf{R}^{2 d}\right)$ with

$$
m(z, \zeta)=\left(1+\left|z_{1}+(1-t) \zeta_{2}\right|+\left|z_{2}-t \zeta_{1}\right|\right)^{s}
$$

or with

$$
m(z, \zeta)=\left(1+\left|z_{1}-t \zeta_{2}\right|+\left|z_{2}+(1-t) \zeta_{1}\right|\right)^{s},
$$

then $\sigma_{t}(X, D) \in C_{p}$.

We will also prove identifications similar to (1.3) for the modulation spaces $M_{m}^{2,2}\left(\mathbf{R}^{2 d}\right)$ of Theorem 1.2. In order to state the results, we use the following notation. We write the partial Fourier transforms of functions $\tau$ on $\mathbf{R}^{2 d}$ as

$$
\begin{aligned}
& F_{1} \tau\left(\zeta_{1}, z_{2}\right)=\int_{\mathbf{R}^{d}} \tau\left(z_{1}, z_{2}\right) e^{-2 \pi i z_{1} \zeta_{1}} d z_{1}, \\
& F_{2} \tau\left(z_{1}, \zeta_{2}\right)=\int_{\mathbf{R}^{d}} \tau\left(z_{1}, z_{2}\right) e^{-2 \pi i z_{2} \zeta_{2}} d z_{2} .
\end{aligned}
$$

For functions $\sigma$ on $\mathbf{R}^{2 d}$ and for $t \in \mathbf{R}$, we define

$$
\tau_{t}\left(z_{1}, z_{2}\right)=e^{2 \pi i t z_{1} z_{2}} \sigma\left(z_{1}, z_{2}\right)
$$

Then we have the following theorem.

TheOREm 1.3. Let $\sigma \in L^{2}\left(\mathbf{R}^{2 d}\right)$, and let $s$ be a nonnegative real number. 
(a) If $m(z, \zeta)=\left(1+\left|z_{1}+t \zeta_{2}\right|\right)^{s}$ with $t \neq 0$, then

$$
\|\sigma\|_{M_{m}^{2,2}} \approx\left\|\left(1+\left|\zeta_{2}\right|\right)^{s} F_{2} \tau_{1 / t}\left(z_{1}, \zeta_{2}\right)\right\|_{L_{z_{1}, \zeta_{2}}^{2}} .
$$

(b) If $m(z, \zeta)=\left(1+\left|z_{1}\right|\right)^{s}$, then

$$
\|\sigma\|_{M_{m}^{2,2}} \approx\left\|\left(1+\left|z_{1}\right|\right)^{s} \sigma\left(z_{1}, z_{2}\right)\right\|_{L_{z_{1}, z_{2}}^{2}} .
$$

(c) If $m(z, \zeta)=\left(1+\left|z_{2}+t \zeta_{1}\right|\right)^{s}$ with $t \neq 0$, then

$$
\|\sigma\|_{M_{m}^{2,2}} \approx\left\|\left(1+\left|\zeta_{1}\right|\right)^{s} F_{1} \tau_{1 / t}\left(\zeta_{1}, z_{2}\right)\right\|_{L_{\zeta_{1}, z_{2}}^{2}} .
$$

(d) If $m(z, \zeta)=\left(1+\left|z_{2}\right|\right)^{s}$, then

$$
\|\sigma\|_{M_{m}^{2,2}} \approx\left\|\left(1+\left|z_{2}\right|\right)^{s} \sigma\left(z_{1}, z_{2}\right)\right\|_{L_{z_{1}, z_{2}}^{2}} .
$$

If, for example, $m$ is the weight of (1.4) and if we define

$$
\begin{aligned}
& m_{1}(z, \zeta)=\left(1+\left|z_{1}+(1-t) \zeta_{2}\right|\right)^{s}, \\
& m_{2}(z, \zeta)=\left(1+\left|z_{2}-t \zeta_{1}\right|\right)^{s}
\end{aligned}
$$

then obviously $m(z, \zeta) \approx m_{1}(z, \zeta)+m_{2}(z, \zeta)$, and hence,

$$
\|\sigma\|_{M_{m}^{2,2}} \approx\|\sigma\|_{M_{m_{1}}^{2,2}}+\|\sigma\|_{M_{m_{2}}^{2,2}} .
$$

This simple fact combined with Theorem 1.3 will give full identifications of the modulation spaces of Theorem 1.2. For example, for the weight $m(z, \zeta)=$ $\left(1+\left|z_{1}+\zeta_{2}\right|+\left|z_{2}\right|\right)^{s}$, which is the $m$ of (1.4) with $t=0$, we have

$$
\|\sigma\|_{M_{m}^{2,2}} \approx\left\|\left(1+\left|\zeta_{2}\right|\right)^{s} F_{2} \tau_{1}\left(z_{1}, \zeta_{2}\right)\right\|_{L_{z_{1}, \zeta_{2}}^{2}}+\left\|\left(1+\left|z_{2}\right|\right)^{s} \sigma\left(z_{1}, z_{2}\right)\right\|_{L_{z_{1}, z_{2}}^{2}} .
$$

Gröchenig and Heil [9] and Heil, Ramanathan, and Topiwala [12] proved Theorem A by constructing finite-rank operators that approximate the pseudodifferential operators. In fact, the argument of [9] and [12] can be modified to give a proof of Theorem 1.2 (see Section 5 below). In the present paper, we will give a different method to prove Theorem 1.2, which is another main purpose of this paper. Our method is based on a modified form of McCarthy's lemma, which characterizes the quasi-norm of $C_{p}$ (see Lemma 2.3), and our argument consists of a direct estimate of the $L^{2}$-norms of functions.

Using the same method, we also prove the following theorem. 
Theorem 1.4. Let $0<p \leq 2$, and let $t \in \mathbf{R}$. If $\sigma \in M^{p, p}\left(\mathbf{R}^{2 d}\right)$, then $\sigma_{t}(X, D) \in C_{p}$.

The case $1 \leq p \leq 2$ of this theorem has already been proved by Gröchenig and Heil [10]. The case $0<p<1$ does not seem to have appeared in the literature.

Finally, we note that Theorems 1.2 and 1.4 are mutually independent; that is, Theorem 1.2 does not cover Theorem 1.4, and Theorem 1.4 does not cover Theorem 1.2. This can be seen from the following two facts. First, if $0<p<2$ and $s>0$, then $M^{p, p}\left(\mathbf{R}^{2 d}\right) \hookrightarrow M_{m}^{2,2}\left(\mathbf{R}^{2 d}\right)$, where $m$ is one of the weights of Theorem 1.2. Second, if $0<p \leq 1, s<(4 d / p)-2 d$, and $m$ is one of the weights of Theorem 1.2 , then $M_{m}^{2,2}\left(\mathbf{R}^{2 d}\right) \hookrightarrow M^{p, p}\left(\mathbf{R}^{2 d}\right)$. The first fact is elementary, and the proof is left to reader. The second fact is proved by Gröchenig [6, Proposition 3] for the case $p=1$. That proof can be generalized to the case $0<p \leq 1$ without essential change.

\section{Notation}

We write $\mathcal{S}\left(\mathbf{R}^{n}\right)$ to denote the Schwartz space of all complex-valued rapidly decreasing infinitely differentiable functions on $\mathbf{R}^{n}$, and we write $\mathcal{S}^{\prime}\left(\mathbf{R}^{n}\right)$ to denote the space of tempered distributions on $\mathbf{R}^{n}$, that is, the topological dual of $\mathcal{S}\left(\mathbf{R}^{n}\right)$. We define

$$
\|f\|_{L^{p}}=\left(\int_{\mathbf{R}^{n}}|f(t)|^{p} d t\right)^{1 / p}
$$

for $0<p<\infty$ and $\|f\|_{L^{\infty}}=\operatorname{ess}_{\sup _{t \in \mathbf{R}^{n}}}|f(t)|$. We use the pairing $\langle f, g\rangle$ between $f \in \mathcal{S}^{\prime}\left(\mathbf{R}^{n}\right)$ and $g \in \mathcal{S}\left(\mathbf{R}^{n}\right)$ in a manner consistent with the inner product

$$
\langle f, g\rangle=\int_{\mathbf{R}^{n}} f(t) \overline{g(t)} d t
$$

on $L^{2}\left(\mathbf{R}^{n}\right)$. For a function $f$ on $\mathbf{R}^{n}$, the translation and the modulation operators are defined by

$$
T_{x} f(t)=f(t-x) \quad \text { and } \quad M_{\omega} f(t)=e^{2 \pi i \omega t} f(t), \quad x, \omega \in \mathbf{R}^{n}
$$

respectively. We note the following:

$$
\left(T_{x} f\right)^{\wedge}=M_{-x} \widehat{f}, \quad\left(M_{\omega} f\right)^{\wedge}=T_{\omega} \widehat{f}, \quad T_{x} M_{\omega}=e^{-2 \pi i x \omega} M_{\omega} T_{x} .
$$

We define $\tilde{f}$ by $\tilde{f}(t)=\overline{f(-t)}$. For $x \in \mathbf{R}^{n}$, we write $\langle x\rangle=\left(1+|x|^{2}\right)^{1 / 2}$. 
For nonnegative functions $F$ and $G$ on a set $X$, we write $F(x) \lesssim G(x)$ $(x \in X)$ if there exists a positive constant $C$ independent of $x \in X$ such that $F(x) \leq C G(x)$ for all $x \in X$. We write $F(x) \approx G(x)(x \in X)$ if $F(x) \lesssim G(x)$ $(x \in X)$ and $G(x) \lesssim F(x)(x \in X)$. We omit to write $(x \in X)$ if the variable $x$ and the domain $X$ are obviously recognized from the context. We also use the notation $G(x) \gtrsim F(x)$ in the same meaning as $F(x) \lesssim G(x)$.

\section{$\S 2$. A modification of McCarthy's lemma}

We first recall the definition of frames for $L^{2}\left(\mathbf{R}^{d}\right)$.

Definition 2.1. A sequence $\left\{f_{\nu}\right\}_{\nu=1}^{\infty}$ in $L^{2}\left(\mathbf{R}^{n}\right)$ is called a frame for $L^{2}\left(\mathbf{R}^{n}\right)$ if there exist constants $A, B>0$ such that

$$
A\|f\|_{L^{2}}^{2} \leq \sum_{\nu=1}^{\infty}\left|\left\langle f, f_{\nu}\right\rangle\right|^{2} \leq B\|f\|_{L^{2}}^{2}, \quad f \in L^{2}\left(\mathbf{R}^{n}\right) .
$$

An orthonormal basis is a special case of the frame. In the proofs of the main results, we will use the Gabor frame $\left\{g_{m n}\right\}_{m, n \in \mathbf{Z}^{d}}$ given in the following example.

EXAMPLE 2.2. Let $g$ be a function in $C_{0}^{\infty}\left(\mathbf{R}^{d}\right)$ such that $\sup g \subset[-1,1]^{d}$ and $\sum_{m \in \mathbf{Z}^{d}}|g(x-m)|^{2}=1$ for all $x \in \mathbf{R}^{d}$. If we define

$$
g_{m n}(x)=M_{\frac{n}{2}} T_{m} g(x)=g(x-m) e^{\pi i n x}, \quad m, n \in \mathbf{Z}^{d},
$$

then the family $\left\{g_{m n}\right\}_{m, n \in \mathbf{Z}^{d}}$ is a frame for $L^{2}\left(\mathbf{R}^{d}\right)$, which satisfies (2.1) with $A=B=1$ (see, e.g., [7, Theorem 6.4.1]).

To estimate the quasi-norm on $C_{p}$, we use the following lemma, which is a modification of McCarthy's lemma (see [15, Lemma 2.2]).

LEMMA 2.3. Let $T$ be a compact operator on $L^{2}\left(\mathbf{R}^{d}\right)$.

(i) If $0<p \leq 2$, then

$$
\|T\|_{C_{p}} \approx \inf \left(\sum_{\nu=1}^{\infty}\left\|T f_{\nu}\right\|_{L^{2}}^{p}\right)^{1 / p}
$$

(ii) If $2 \leq p<\infty$, then

$$
\|T\|_{C_{p}} \approx \sup \left(\sum_{\nu=1}^{\infty}\left\|T f_{\nu}\right\|_{L^{2}}^{p}\right)^{1 / p} .
$$

In (i) and (ii), we take the infimum or supremum over all frames $\left\{f_{\nu}\right\}_{\nu=1}^{\infty}$ for $L^{2}\left(\mathbf{R}^{d}\right)$ satisfying (2.1) for fixed $A$ and $B$. 
McCarthy [15, Lemma 2.2] gave this lemma using orthonormal bases instead of frames, in which case the relations hold with equality in place of $\approx$. The following proof is a modification of the argument given in the book of Komatsu [14, Proposition 8.5].

Proof of Lemma 2.3.

(i) By the Schmidt representation, $T$ can be written as

$$
T f=\sum_{j=1}^{\infty} \lambda_{j}\left\langle f, e_{j}\right\rangle e_{j}^{\prime}, \quad f \in L^{2}\left(\mathbf{R}^{d}\right),
$$

where $\left\{e_{j}\right\}_{j=1}^{\infty}$ and $\left\{e_{j}^{\prime}\right\}_{j=1}^{\infty}$ are orthonormal systems and $\lambda_{j}=s_{j}(T)$ (see, e.g., [16, Proposition 16.3]).

Let $\left\{f_{\nu}\right\}$ be an arbitrary frame for $L^{2}\left(\mathbf{R}^{d}\right)$ satisfying (2.1). Since $0<p \leq$ 2 , the function $t^{p / 2}$ is concave. Therefore, for any sequence $\left\{\alpha_{j}\right\}_{j=1}^{\infty}$ with $\alpha_{j} \geq 0$ and $\sum_{j=1}^{\infty} \alpha_{j} \leq B$, we have

$$
\left(\sum_{j=1}^{\infty} \frac{\alpha_{j}}{B} \lambda_{j}^{2}\right)^{p / 2} \geq \sum_{j=1}^{\infty} \frac{\alpha_{j}}{B} \lambda_{j}^{p} .
$$

Taking $\alpha_{j}=\left|\left\langle f_{\nu}, e_{j}\right\rangle\right|^{2}$, we have $\sum_{j} \alpha_{j}=\sum_{j}\left|\left\langle f_{\nu}, e_{j}\right\rangle\right|^{2} \leq\left\|f_{\nu}\right\|_{L^{2}}^{2} \leq B$ and

$$
\begin{aligned}
\sum_{\nu}\left\|T f_{\nu}\right\|_{L^{2}}^{p} & =\sum_{\nu}\left(\sum_{j=1}^{\infty}\left|\left\langle f_{\nu}, e_{j}\right\rangle\right|^{2} \lambda_{j}^{2}\right)^{p / 2} \geq B^{(p / 2)-1} \sum_{\nu} \sum_{j=1}^{\infty}\left|\left\langle f_{\nu}, e_{j}\right\rangle\right|^{2} \lambda_{j}^{p} \\
& \geq A B^{(p / 2)-1} \sum_{j=1}^{\infty} \lambda_{j}^{p} .
\end{aligned}
$$

Thus,

$$
\begin{aligned}
\inf \left(\sum_{\nu}\left\|T f_{\nu}\right\|_{L^{2}}^{p}\right)^{1 / p} & \geq A^{1 / p} B^{(1 / 2)-(1 / p)}\left(\sum_{j=1}^{\infty} \lambda_{j}^{p}\right)^{1 / p} \\
& =A^{1 / p} B^{(1 / 2)-(1 / p)}\|T\|_{C_{p}} .
\end{aligned}
$$

On the other hand, let $\left\{\widetilde{e}_{\nu}\right\}$ be an orthonormal basis for $L^{2}\left(\mathbf{R}^{d}\right)$ containing $\left\{e_{j}\right\}$, and put $f_{\nu}=A^{1 / 2} \widetilde{e}_{\nu}$. Then $\left\{f_{\nu}\right\}$ is a frame for $L^{2}\left(\mathbf{R}^{d}\right)$ satisfying (2.1). Since $T f_{\nu}=\sum_{j} \lambda_{j}\left\langle A^{1 / 2} \widetilde{e}_{\nu}, e_{j}\right\rangle e_{j}^{\prime}=\lambda_{j(\nu)} A^{1 / 2} e_{j(\nu)}^{\prime}$ if $\widetilde{e}_{\nu}=e_{j(\nu)}$ is in $\left\{e_{j}\right\}$, and $=0$ if $\widetilde{e}_{\nu} \notin\left\{e_{j}\right\}$, we have

$$
\sum_{\nu}\left\|T f_{\nu}\right\|_{L^{2}}^{p}=A^{p / 2} \sum_{j=1}^{\infty} \lambda_{j}^{p} .
$$


Therefore,

$$
\inf \left(\sum_{\nu}\left\|T f_{\nu}\right\|_{L^{2}}^{p}\right)^{1 / p} \leq A^{1 / 2}\left(\sum_{j=1}^{\infty} \lambda_{j}^{p}\right)^{1 / p}=A^{1 / 2}\|T\|_{C_{p}} .
$$

(ii) If $2 \leq p<\infty$, then we can actually prove the equality

$$
\sup \left(\sum_{\nu}\left\|T f_{\nu}\right\|_{L^{2}}^{p}\right)^{1 / p}=B^{1 / 2}\|T\|_{C^{p}}
$$

In fact, for $2 \leq p<\infty$, the function $t^{p / 2}$ is convex, and the inequality reverse to $(2.2)$ holds. Hence, we can prove the inequality

$$
\sup \left(\sum_{\nu}\left\|T f_{\nu}\right\|_{L^{2}}^{p}\right)^{1 / p} \leq B^{1 / 2}\|T\|_{C^{p}}
$$

by the same argument as in the first half of (i). The converse inequality can be seen by the use of the frame $f_{\nu}=B^{1 / 2} \widetilde{e}_{\nu}$ with $\widetilde{e}_{\nu}$ being the same as in (i).

\section{$\S 3$. Proof of Theorems 1.2 and 1.4}

Before we prove Theorems 1.2 and 1.4, we recall some basic properties of modulation spaces $M_{m}^{p, q}\left(\mathbf{R}^{n}\right)$. (For the proofs, we refer to [3], [5], [7], [13], or [17].) The space $M_{m}^{p, q}\left(\mathbf{R}^{n}\right)$ is a quasi-Banach space. If $0<p, q<\infty$, then $\mathcal{S}\left(\mathbf{R}^{n}\right)$ is dense in $M_{m}^{p, q}\left(\mathbf{R}^{n}\right)$. If $0<p_{1} \leq p_{2} \leq \infty, 0<q_{1} \leq q_{2} \leq \infty$, and $m_{2} \lesssim m_{1}$, then $M_{m_{1}}^{p_{1}, q_{1}}\left(\mathbf{R}^{n}\right) \hookrightarrow M_{m_{2}}^{p_{2}, q_{2}}\left(\mathbf{R}^{n}\right)$. The weight functions $m$ of Theorem A and Theorem 1.2 satisfy condition (1.1) with $n=2 d$.

The following lemma will be used to represent $\sigma(x, D)$ as a superposition of "elementary operators."

Lemma 3.1 ([7, Corollary 11.2.7]). If $\varphi, \psi, f \in \mathcal{S}\left(\mathbf{R}^{n}\right)$ and if $\langle\varphi, \widetilde{\psi}\rangle \neq 0$, then

$$
f(t)=\frac{1}{\langle\varphi, \widetilde{\psi}\rangle} \int_{\mathbf{R}^{2 n}} f * M_{\zeta} \varphi(z) T_{z} M_{\zeta} \psi(t) d z d \zeta, \quad t \in \mathbf{R}^{n},
$$

where the function

$$
(z, \zeta, t) \mapsto f * M_{\zeta} \varphi(z) T_{z} M_{\zeta} \psi(t)
$$

is in the class $\mathcal{S}$ on $\mathbf{R}^{3 n}=\mathbf{R}_{z}^{n} \times \mathbf{R}_{\zeta}^{n} \times \mathbf{R}_{t}^{n}$. 
Proof of Theorem 1.2. PART I. We prove the result for the weight (1.4) with $t=0$. Notice that $M_{m}^{2,2}\left(\mathbf{R}^{2 d}\right) \subset L^{2}\left(\mathbf{R}^{2 d}\right)$ and that the pseudodifferential operators $\sigma(X, D)$ with symbols in $L^{2}\left(\mathbf{R}^{2 d}\right)$ are of Hilbert-Schmidt class. Hence, $\sigma(X, D)$ with $\sigma \in M_{m}^{2,2}\left(\mathbf{R}^{2 d}\right)$ are in the Hilbert-Schmidt class and, in particular, compact.

Let $\left\{g_{m n}\right\}$ be the frame given in Example 2.2. By virtue of Lemma 2.3(i), the claim of Theorem 1.2 for $\sigma(X, D)$ follows from the inequality

$$
\left(\sum_{m, n \in \mathbf{Z}^{d}}\left\|\sigma(X, D) g_{m n}\right\|_{L^{2}}^{p}\right)^{1 / p} \leq C\|\sigma\|_{M_{m}^{2,2}}
$$

Since $\mathcal{S}\left(\mathbf{R}^{2 d}\right)$ is dense in $M_{m}^{2,2}\left(\mathbf{R}^{2 d}\right)$, it is sufficient to prove this estimate for $\sigma \in \mathcal{S}\left(\mathbf{R}^{2 d}\right)$. Thus, in the rest of the argument, we assume that $\sigma \in \mathcal{S}\left(\mathbf{R}^{2 d}\right)$.

Take $\Phi, \Psi \in \mathcal{S}\left(\mathbf{R}^{2 d}\right)$ such that $\langle\Phi, \widetilde{\Psi}\rangle \neq 0$. By Lemma 3.1 with $n=2 d$, we have

$$
\sigma(x, \omega)=\frac{1}{\langle\Phi, \widetilde{\Psi}\rangle} \int_{\mathbf{R}^{4 d}} \sigma * M_{\zeta} \Phi(z) T_{z} M_{\zeta} \Psi(x, \omega) d z d \zeta, \quad x, \omega \in \mathbf{R}^{d} .
$$

Let $f \in \mathcal{S}\left(\mathbf{R}^{d}\right)$. The above formula yields

$$
\begin{aligned}
& \sigma(X, D) f(x)=\frac{1}{\langle\Phi, \widetilde{\Psi}\rangle} \int_{\mathbf{R}^{5 d}} \sigma * M_{\zeta} \Phi(z) T_{z} M_{\zeta} \Psi(x, \omega) \widehat{f}(\omega) e^{2 \pi i x \omega} d z d \zeta d \omega, \\
& x \in \mathbf{R}^{d}
\end{aligned}
$$

Notice that the above integrand is a function in the class $\mathcal{S}$ on $\mathbf{R}^{6 d}=\mathbf{R}_{z}^{2 d} \times$ $\mathbf{R}_{\zeta}^{2 d} \times \mathbf{R}_{\omega}^{d} \times \mathbf{R}_{x}^{d}$. Thus, we obtain

$$
\|\sigma(X, D) f\|_{L^{2}}^{2}
$$

$$
\begin{aligned}
= & \frac{1}{|\langle\Phi, \widetilde{\Psi}\rangle|^{2}} \int_{\mathbf{R}^{11 d}} \sigma * M_{\zeta} \Phi(z) T_{z} M_{\zeta} \Psi(x, \omega) \widehat{f}(\omega) e^{2 \pi i x \omega} \\
& \times \overline{\sigma * M_{\zeta^{\prime}} \Phi\left(z^{\prime}\right)} \overline{T_{z^{\prime}} M_{\zeta^{\prime}} \Psi\left(x, \omega^{\prime}\right)} \overline{\widehat{f}\left(\omega^{\prime}\right)} e^{-2 \pi i x \omega^{\prime}} d z d \zeta d \omega d z^{\prime} d \zeta^{\prime} d \omega^{\prime} d x .
\end{aligned}
$$

The integrand of (3.1) is a function in the class $\mathcal{S}$ on $\mathbf{R}^{11 d}=\mathbf{R}_{z}^{2 d} \times \mathbf{R}_{\zeta}^{2 d} \times$ $\mathbf{R}_{\omega}^{d} \times \mathbf{R}_{z^{\prime}}^{2 d} \times \mathbf{R}_{\zeta^{\prime}}^{2 d} \times \mathbf{R}_{\omega^{\prime}}^{d} \times \mathbf{R}_{x}^{d}$, and hence we can freely change the order of integration.

We simply write

$$
\alpha(z, \zeta)=\sigma * M_{\zeta} \Phi(z)
$$


and apply (3.1) to $f=g_{m n}$. We have

$$
\widehat{g_{m n}}(\omega)=\widehat{g}(\omega-n / 2) e^{-2 \pi i m(\omega-n / 2)}
$$

and

$$
\begin{aligned}
& T_{z} M_{\zeta} \Psi(x, \omega) e^{2 \pi i x \omega} \\
& \quad=\Theta\left(x-z_{1}, \omega-z_{2}\right) e^{2 \pi i x\left(z_{2}+\zeta_{1}\right)} e^{2 \pi i \omega\left(z_{1}+\zeta_{2}\right)} e^{2 \pi i\left(-z_{1} z_{2}-z_{1} \zeta_{1}-z_{2} \zeta_{2}\right)},
\end{aligned}
$$

where $\Theta(x, \omega)=\Psi(x, \omega) e^{2 \pi i x \omega}$. With these expressions, (3.1) can be written as

$$
\begin{aligned}
\left\|\sigma(X, D) g_{m n}\right\|_{L^{2}}^{2} \\
=\frac{1}{|\langle\Phi, \widetilde{\Psi}\rangle|^{2}} \int_{\mathbf{R}^{11 d}} e^{2 \pi i\left(-z_{1} z_{2}-z_{1} \zeta_{1}-z_{2} \zeta_{2}\right)} \\
\quad \times e^{2 \pi i\left(z_{1}^{\prime} z_{2}^{\prime}+z_{1}^{\prime} \zeta_{1}^{\prime}+z_{2}^{\prime} \zeta_{2}^{\prime}\right)} \alpha(z, \zeta) \overline{\alpha\left(z^{\prime}, \zeta^{\prime}\right)} e^{2 \pi i x\left(z_{2}-z_{2}^{\prime}+\zeta_{1}-\zeta_{1}^{\prime}\right)} \\
\quad \times e^{2 \pi i \omega\left(z_{1}+\zeta_{2}-m\right)} e^{2 \pi i \omega^{\prime}\left(-z_{1}^{\prime}-\zeta_{2}^{\prime}+m\right)} \Theta\left(x-z_{1}, \omega-z_{2}\right) \overline{\Theta\left(x-z_{1}^{\prime}, \omega^{\prime}-z_{2}^{\prime}\right)} \\
\quad \times \widehat{g}(\omega-n / 2) \overline{\widehat{g}\left(\omega^{\prime}-n / 2\right)} d z d \zeta d \omega d z^{\prime} d \zeta^{\prime} d \omega^{\prime} d x .
\end{aligned}
$$

In the integral of (3.2), we first take the integration with respect to $\left(x, \omega, \omega^{\prime}\right)$. Consider the integral

$$
\begin{aligned}
\int_{\mathbf{R}^{3 d}} & e^{2 \pi i x\left(z_{2}-z_{2}^{\prime}+\zeta_{1}-\zeta_{1}^{\prime}\right)} e^{2 \pi i \omega\left(z_{1}+\zeta_{2}-m\right)} e^{2 \pi i \omega^{\prime}\left(-z_{1}^{\prime}-\zeta_{2}^{\prime}+m\right)} \\
& \times \Theta\left(x-z_{1}, \omega-z_{2}\right) \overline{\Theta\left(x-z_{1}^{\prime}, \omega^{\prime}-z_{2}^{\prime}\right)} \\
& \times \widehat{g}(\omega-n / 2) \overline{\widehat{g}\left(\omega^{\prime}-n / 2\right)} d x d \omega d \omega^{\prime}
\end{aligned}
$$

This is the inverse Fourier transform of the function

$$
\begin{aligned}
\left(x, \omega, \omega^{\prime}\right) \mapsto & \Theta\left(x-z_{1}, \omega-z_{2}\right) \overline{\Theta\left(x-z_{1}^{\prime}, \omega^{\prime}-z_{2}^{\prime}\right)} \\
& \times \widehat{g}(\omega-n / 2) \overline{\widehat{g}\left(\omega^{\prime}-n / 2\right)}
\end{aligned}
$$

evaluated at $\left(z_{2}-z_{2}^{\prime}+\zeta_{1}-\zeta_{1}^{\prime}, z_{1}+\zeta_{2}-m,-z_{1}^{\prime}-\zeta_{2}^{\prime}+m\right)$. Since $\Theta$ and $\widehat{g}$ are in the class $\mathcal{S}$, we see that the absolute value of the function (3.4) is majorized by a constant times

$$
\left\langle x-z_{1}\right\rangle^{-N}\left\langle\omega-z_{2}\right\rangle^{-N}\left\langle\omega^{\prime}-z_{2}^{\prime}\right\rangle^{-N}\left\langle z_{1}-z_{1}^{\prime}\right\rangle^{-N}\left\langle z_{2}-n / 2\right\rangle^{-N}\left\langle z_{2}^{\prime}-n / 2\right\rangle^{-N}
$$


where $N$ can be taken as arbitrarily large. The same estimate also holds for every derivative of (3.4). Hence, by integration by parts, we see that the absolute value of integral (3.3) is majorized by a constant times

$$
\begin{aligned}
\left\langle z_{1}-\right. & \left.z_{1}^{\prime}\right\rangle^{-N}\left\langle z_{2}-n / 2\right\rangle^{-N}\left\langle z_{2}^{\prime}-n / 2\right\rangle^{-N} \\
& \times\left\langle z_{2}-z_{2}^{\prime}+\zeta_{1}-\zeta_{1}^{\prime}\right\rangle^{-N}\left\langle z_{1}+\zeta_{2}-m\right\rangle^{-N}\left\langle-z_{1}^{\prime}-\zeta_{2}^{\prime}+m\right\rangle^{-N}
\end{aligned}
$$

Combining the last estimate with (3.2), we obtain

$$
\begin{aligned}
& \left\|\sigma(X, D) g_{m n}\right\|_{L^{2}}^{2} \\
& \leq c \int_{\mathbf{R}^{8 d}}|\alpha(z, \zeta)|\left|\alpha\left(z^{\prime}, \zeta^{\prime}\right)\right|\left\langle z_{1}-z_{1}^{\prime}\right\rangle^{-N}\left\langle z_{2}-n / 2\right\rangle^{-N} \\
& \times\left\langle z_{2}^{\prime}-n / 2\right\rangle^{-N}\left\langle z_{2}-z_{2}^{\prime}+\zeta_{1}-\zeta_{1}^{\prime}\right\rangle^{-N}\left\langle z_{1}+\zeta_{2}-m\right\rangle^{-N} \\
& \times\left\langle-z_{1}^{\prime}-\zeta_{2}^{\prime}+m\right\rangle^{-N} d z d \zeta d z^{\prime} d \zeta^{\prime} \\
& =(*) .
\end{aligned}
$$

We use the inequality $|\alpha(z, \zeta)|\left|\alpha\left(z^{\prime}, \zeta^{\prime}\right)\right| \leq 2^{-1}\left(|\alpha(z, \zeta)|^{2}+\left|\alpha\left(z^{\prime}, \zeta^{\prime}\right)\right|^{2}\right)$ and use the symmetry of the variables $(z, \zeta)$ and $\left(z^{\prime}, \zeta^{\prime}\right)$ to see that

$$
\begin{aligned}
&(*) \leq c \int_{\mathbf{R}^{8 d}}|\alpha(z, \zeta)|^{2}\left\langle z_{1}-z_{1}^{\prime}\right\rangle^{-N}\left\langle z_{2}-n / 2\right\rangle^{-N} \\
& \times\left\langle z_{2}^{\prime}-n / 2\right\rangle^{-N}\left\langle z_{2}-z_{2}^{\prime}+\zeta_{1}-\zeta_{1}^{\prime}\right\rangle^{-N} \\
& \times\left\langle z_{1}+\zeta_{2}-m\right\rangle^{-N}\left\langle-z_{1}^{\prime}-\zeta_{2}^{\prime}+m\right\rangle^{-N} d z d \zeta d z^{\prime} d \zeta^{\prime} \\
&=c \int_{\mathbf{R}^{6 d}}|\alpha(z, \zeta)|^{2}\left\langle z_{1}-z_{1}^{\prime}\right\rangle^{-N}\left\langle z_{2}-n / 2\right\rangle^{-N} \quad \times\left\langle z_{2}^{\prime}-n / 2\right\rangle^{-N}\left\langle z_{1}+\zeta_{2}-m\right\rangle^{-N} d z d \zeta d z^{\prime} \\
&=c \int_{\mathbf{R}^{4 d}}|\alpha(z, \zeta)|^{2}\left\langle z_{1}+\zeta_{2}-m\right\rangle^{-N}\left\langle z_{2}-n / 2\right\rangle^{-N} d z d \zeta
\end{aligned}
$$

Thus, we have proved the estimate

$$
\begin{aligned}
\left\|\sigma(X, D) g_{m n}\right\|_{L^{2}}^{2} \leq c \int_{\mathbf{R}^{4 d}} & |\alpha(z, \zeta)|^{2}\left\langle z_{1}+\zeta_{2}-m\right\rangle^{-N} \\
& \times\left\langle z_{2}-n / 2\right\rangle^{-N} d z d \zeta .
\end{aligned}
$$


Let $q$ be the real number defined by $p / 2+1 / q=1$. Then (3.5) and Hölder's inequality give

$$
\begin{aligned}
& \sum_{m, n \in \mathbf{Z}^{d}}\left\|\sigma(X, D) g_{m n}\right\|_{L^{2}}^{p} \\
& \leq c \sum_{m, n \in \mathbf{Z}^{d}}\left(\int_{\mathbf{R}^{4 d}}|\alpha(z, \zeta)|^{2}\left\langle z_{1}+\zeta_{2}-m\right\rangle^{-N}\left\langle z_{2}-\frac{n}{2}\right\rangle^{-N} d z d \zeta\right)^{p / 2} \\
& \leq c\left(\sum_{m, n \in \mathbf{Z}^{d}}(1+|m|+|n|)^{2 s} \int_{\mathbf{R}^{4 d}}|\alpha(z, \zeta)|^{2}\left\langle z_{1}+\zeta_{2}-m\right\rangle^{-N}\right. \\
&\left.\times\left(\sum_{m, n \in \mathbf{Z}^{d}}(1+|m|+|n|)^{-s p q}\right)^{1 / q} \times\left\langle z_{2}-\frac{n}{2}\right\rangle^{-N} d z d \zeta\right)^{p / 2} \\
&= c\left(\sum_{m, n \in \mathbf{Z}^{d}}(1+|m|+|n|)^{2 s} \int_{\mathbf{R}^{4 d}}|\alpha(z, \zeta)|^{2}\left\langle z_{1}+\zeta_{2}-m\right\rangle^{-N}\right. \\
&=(* *), \\
&\left.\times\left\langle z_{2}-\frac{n}{2}\right\rangle^{-N} d z d \zeta\right)^{p / 2}
\end{aligned}
$$

where the first $=$ holds because $s p q>2 d$ by our assumption that $s>2 d /$ $p-d$. Since, for sufficiently large $N$,

$$
\begin{aligned}
& \sum_{m, n \in \mathbf{Z}^{d}}(1+|m|+|n|)^{2 s}\left\langle z_{1}+\zeta_{2}-m\right\rangle^{-N}\left\langle z_{2}-\frac{n}{2}\right\rangle^{-N} \\
& \quad \approx \sum_{n \in \mathbf{Z}^{d}}\left(1+\left|z_{1}+\zeta_{2}\right|+|n|\right)^{2 s}\left\langle z_{2}-\frac{n}{2}\right\rangle^{-N} \approx\left(1+\left|z_{1}+\zeta_{2}\right|+\left|z_{2}\right|\right)^{2 s},
\end{aligned}
$$

we obtain

$$
(* *) \approx\left(\int_{\mathbf{R}^{4 d}}|\alpha(z, \zeta)|^{2}\left(1+\left|z_{1}+\zeta_{2}\right|+\left|z_{2}\right|\right)^{2 s} d z d \zeta\right)^{p / 2} \approx\|\sigma\|_{M_{m}^{2,2}}^{p},
$$

where $m$ is the weight of (1.4) with $t=0$. Thus, the claim for the weight (1.4) with $t=0$ is proved. 
PART II. We prove the result for the weight (1.5) with $t=0$. To this end, we consider the adjoint operator $\sigma(X, D)^{*}$ of $\sigma(X, D)$. Take $\Phi, \Psi \in \mathcal{S}\left(\mathbf{R}^{2 d}\right)$ such that $\langle\Phi, \widetilde{\Psi}\rangle \neq 0$, and represent $\sigma(X, D)$ as

$$
\sigma(X, D) f(x)=\frac{1}{\langle\Phi, \widetilde{\Psi}\rangle} \int_{\mathbf{R}^{5 d}} \alpha(z, \zeta) T_{z} M_{\zeta} \Psi(x, \omega) \widehat{f}(\omega) e^{2 \pi i x \omega} d z d \zeta d \omega
$$

where $\alpha(z, \zeta)=\sigma * M_{\zeta} \Phi(z)$. Then we have

$$
\begin{aligned}
& \left\langle f, \sigma(X, D)^{*} \psi\right\rangle \\
& =\langle\sigma(X, D) f, \psi\rangle=\int_{\mathbf{R}^{d}} \sigma(X, D) f(x) \overline{\psi(x)} d x \\
& =\frac{1}{\langle\Phi, \widetilde{\Psi}\rangle} \int_{\mathbf{R}^{6 d}} \alpha(z, \zeta) T_{z} M_{\zeta} \Psi(x, \omega) \widehat{f}(\omega) e^{2 \pi i x \omega} \overline{\psi(x)} d z d \zeta d \omega d x \\
& =\frac{1}{\langle\Phi, \widetilde{\Psi}\rangle} \int_{\mathbf{R}^{d}} \widehat{f}(\omega) \int_{\mathbf{R}^{5 d}} \alpha(z, \zeta) T_{z} M_{\zeta} \Psi(x, \omega) e^{2 \pi i x \omega} \overline{\psi(x)} d z d \zeta d x d \omega \\
& =\left\langle f, \overline{\mathcal{F}_{\omega}\left[\frac{1}{\langle\Phi, \widetilde{\Psi}\rangle} \int_{\mathbf{R}^{5 d}} \alpha(z, \zeta) T_{z} M_{\zeta} \Psi(x, \omega) e^{2 \pi i x \omega} \overline{\psi(x)} d z d \zeta d x\right]}\right\rangle,
\end{aligned}
$$

where $\mathcal{F}_{\omega}$ denotes the Fourier transform with respect to the variable $\omega$. Thus,

$$
\overline{\sigma(X, D)^{*} \psi}=\mathcal{F}_{\omega}\left[\frac{1}{\langle\Phi, \widetilde{\Psi}\rangle} \int_{\mathbf{R}^{5 d}} \alpha(z, \zeta) T_{z} M_{\zeta} \Psi(x, \omega) e^{2 \pi i x \omega} \overline{\psi(x)} d z d \zeta d x\right] .
$$

Since $\|\sigma(X, D)\|_{C_{p}}=\left\|\sigma(X, D)^{*}\right\|_{C_{p}}$ (see [2, p. 1092]), we have

$$
\|\sigma(X, D)\|_{C_{p}} \lesssim\left(\sum_{m, n \in \mathbf{Z}^{d}}\left\|\sigma(X, D)^{*} g_{m n}\right\|_{L^{2}}^{p}\right)^{1 / p}
$$

by Lemma 2.3(i), where $\left\{g_{m n}\right\}$ is the frame of Example 2.2. So, we estimate $\left\|\sigma(X, D)^{*} g_{m n}\right\|_{L^{2}}$. Using the Plancherel theorem and (3.6) with $\psi=g_{m n}$, we have

$$
\begin{aligned}
& \left\|\sigma(X, D)^{*} g_{m n}\right\|_{L^{2}}^{2} \\
& \quad \approx\left\|\int_{\mathbf{R}^{5 d}} \alpha(z, \zeta) T_{z} M_{\zeta} \Psi(x, \omega) e^{2 \pi i x \omega} \overline{g_{m n}(x)} d z d \zeta d x\right\|_{L_{\omega}^{2}}^{2}
\end{aligned}
$$




$$
\begin{aligned}
=\int_{\mathbf{R}^{11 d}} & \alpha(z, \zeta) \overline{\alpha\left(z^{\prime}, \zeta^{\prime}\right)} e^{2 \pi i\left(-z_{1} z_{2}-z_{1} \zeta_{1}-z_{2} \zeta_{2}\right)} e^{2 \pi i\left(z_{1}^{\prime} z_{2}^{\prime}+z_{1}^{\prime} \zeta_{1}^{\prime}+z_{2}^{\prime} \zeta_{2}^{\prime}\right)} \\
& \times \Theta\left(x-z_{1}, \omega-z_{2}\right) \overline{\Theta\left(x^{\prime}-z_{1}^{\prime}, \omega-z_{2}^{\prime}\right)} \overline{g(x-m)} \\
& \times g\left(x^{\prime}-m\right) e^{2 \pi i x\left(z_{2}+\zeta_{1}-n / 2\right)} e^{2 \pi i x^{\prime}\left(-z_{2}^{\prime}-\zeta_{1}^{\prime}+n / 2\right)} \\
& \times e^{2 \pi i \omega\left(z_{1}+\zeta_{2}-z_{1}^{\prime}-\zeta_{2}^{\prime}\right)} d z d \zeta d x d z^{\prime} d \zeta^{\prime} d x^{\prime} d \omega \\
=(*), &
\end{aligned}
$$

where $\Theta(x, \omega)=\Psi(x, \omega) e^{2 \pi i x \omega}$. In the above integral, we first take the integration with respect to $\left(x, x^{\prime}, \omega\right)$. As in the proof of Part I, we obtain

$$
\begin{aligned}
\mid \int_{\mathbf{R}^{3 d}} & e^{2 \pi i x\left(z_{2}+\zeta_{1}-n / 2\right)} e^{2 \pi i x^{\prime}\left(-z_{2}^{\prime}-\zeta_{1}^{\prime}+n / 2\right)} e^{2 \pi i \omega\left(z_{1}+\zeta_{2}-z_{1}^{\prime}-\zeta_{2}^{\prime}\right)} \\
& \times \Theta\left(x-z_{1}, \omega-z_{2}\right) \overline{\Theta\left(x^{\prime}-z_{1}^{\prime}, \omega-z_{2}^{\prime}\right)} \overline{g(x-m)} g\left(x^{\prime}-m\right) d x d x^{\prime} d \omega \mid \\
\lesssim & \left\langle z_{1}-m\right\rangle^{-N}\left\langle z_{1}^{\prime}-m\right\rangle^{-N}\left\langle z_{2}-z_{2}^{\prime}\right\rangle^{-N} \\
& \times\left\langle z_{2}+\zeta_{1}-n / 2\right\rangle^{-N}\left\langle-z_{2}^{\prime}-\zeta_{1}^{\prime}+n / 2\right\rangle^{-N}\left\langle z_{1}+\zeta_{2}-z_{1}^{\prime}-\zeta_{2}^{\prime}\right\rangle^{-N}
\end{aligned}
$$

where $N$ can be taken arbitrarily large. Thus,

$$
\begin{aligned}
&(*) \lesssim \int_{\mathbf{R}^{8 d}}|\alpha(z, \zeta)|\left|\alpha\left(z^{\prime}, \zeta^{\prime}\right)\right|\left\langle z_{1}-m\right\rangle^{-N}\left\langle z_{1}^{\prime}-m\right\rangle^{-N}\left\langle z_{2}-z_{2}^{\prime}\right\rangle^{-N} \\
& \times\left\langle z_{2}+\zeta_{1}-n / 2\right\rangle^{-N}\left\langle-z_{2}^{\prime}-\zeta_{1}^{\prime}+n / 2\right\rangle^{-N} \\
& \times\left\langle z_{1}+\zeta_{2}-z_{1}^{\prime}-\zeta_{2}^{\prime}\right\rangle^{-N} d z d \zeta d z^{\prime} d \zeta^{\prime} \\
& \lesssim \int_{\mathbf{R}^{8 d}}|\alpha(z, \zeta)|^{2}\left\langle z_{1}-m\right\rangle^{-N}\left\langle z_{1}^{\prime}-m\right\rangle^{-N}\left\langle z_{2}-z_{2}^{\prime}\right\rangle^{-N} \\
& \quad \times\left\langle z_{2}+\zeta_{1}-n / 2\right\rangle^{-N}\left\langle-z_{2}^{\prime}-\zeta_{1}^{\prime}+n / 2\right\rangle^{-N} \\
& \quad \times\left\langle z_{1}+\zeta_{2}-z_{1}^{\prime}-\zeta_{2}^{\prime}\right\rangle^{-N} d z d \zeta d z^{\prime} d \zeta^{\prime} \\
& \approx \int_{\mathbf{R}^{6 d}}|\alpha(z, \zeta)|^{2}\left\langle z_{1}-m\right\rangle^{-N}\left\langle z_{1}^{\prime}-m\right\rangle^{-N}\left\langle z_{2}-z_{2}^{\prime}\right\rangle{ }^{-N} \\
& \quad \times\left\langle z_{2}+\zeta_{1}-n / 2\right\rangle^{-N} d z d \zeta d z^{\prime} \\
& \approx \int_{\mathbf{R}^{4 d}}|\alpha(z, \zeta)|^{2}\left\langle z_{1}-m\right\rangle^{-N}\left\langle z_{2}+\zeta 1-n / 2\right\rangle^{-N} d z d \zeta
\end{aligned}
$$


Hence, in the same way as in Part I, we obtain

$$
\begin{aligned}
\|\sigma(X, D)\|_{C_{p}}^{p} & \\
& \lesssim \sum_{m, n \in \mathbf{Z}^{d}}\left\|\sigma(X, D)^{*} g_{m n}\right\|_{L^{2}}^{p} \\
& \lesssim \sum_{m, n \in \mathbf{Z}^{d}}\left(\int_{\mathbf{R}^{4 d}}|\alpha(z, \zeta)|^{2}\left\langle z_{1}-m\right\rangle^{-N}\left\langle z_{2}+\zeta_{1}-\frac{n}{2}\right\rangle^{-N} d z d \zeta\right)^{p / 2} \\
& \lesssim\left(\sum_{m, n \in \mathbf{Z}^{d}}(1+|m|+|n|)^{2 s}\right. \\
& \left.\times \int_{\mathbf{R}^{4 d}}|\alpha(z, \zeta)|^{2}\left\langle z_{1}-m\right\rangle^{-N}\left\langle z_{2}+\zeta_{1}-\frac{n}{2}\right\rangle^{-N} d z d \zeta\right)^{p / 2} \\
& \approx\left(\int_{\mathbf{R}^{4 d}}|\alpha(z, \zeta)|^{2}\left(1+\left|z_{1}\right|+\left|z_{2}+\zeta_{1}\right|\right)^{2 s} d z d \zeta\right)^{p / 2} \approx\|\sigma\|_{M_{m}^{2,2}}^{p}
\end{aligned}
$$

where $s>2 d / p-d$ and where $m$ is the weight of (1.5) with $t=0$. This proves the claim for the weight (1.5) with $t=0$.

PART III. Finally, we prove the result for general $t \in \mathbf{R}$. We use the fact that $\sigma_{t}(X, D)$ can be written as $\tau(X, D)$ by a simple transformation of symbols $\sigma \mapsto \tau$. In fact, if we define the symbol $\mathcal{U}_{t} \sigma$ by

$$
\left(\mathcal{U}_{t} \sigma\right)^{\wedge}(\xi, \eta)=e^{2 \pi i t \xi \eta} \widehat{\sigma}(\xi, \eta)
$$

then $\sigma_{t}(X, D)=\left(\mathcal{U}_{t} \sigma\right)(X, D)$. We have

$$
\left(\mathcal{U}_{t} \sigma\right) * M_{\zeta} \Phi(z)=e^{-2 \pi i t \zeta_{1} \zeta_{2}} \sigma * M_{\zeta} \mathcal{U}_{t} \Phi\left(z_{1}+t \zeta_{2}, z_{2}+t \zeta_{1}\right)
$$

(see [9, Lemma 2.1], [7, Corollary 14.5.5]). Hence, the estimate obtained in Part I yields

$$
\begin{aligned}
\left\|\sigma_{t}(X, D)\right\|_{C_{p}} & =\left\|\left(\mathcal{U}_{t} \sigma\right)(X, D)\right\|_{C_{p}} \\
& \lesssim\left(\int_{\mathbf{R}^{4 d}}\left|\left(\mathcal{U}_{t} \sigma\right) * M_{\zeta} \Phi(z)\right|^{2}\left(1+\left|z_{1}+\zeta_{2}\right|+\left|z_{2}\right|\right)^{2 s} d z d \zeta\right)^{1 / 2} \\
= & \left(\int_{\mathbf{R}^{4 d}}\left|\sigma * M_{\zeta} \mathcal{U}_{t} \Phi\left(z_{1}+t \zeta_{2}, z_{2}+t \zeta_{1}\right)\right|^{2}\right. \\
& \left.\times\left(1+\left|z_{1}+\zeta_{2}\right|+\left|z_{2}\right|\right)^{2 s} d z d \zeta\right)^{1 / 2}
\end{aligned}
$$




$$
\begin{aligned}
=\left(\int_{\mathbf{R}^{4 d}} \mid\right. & \left.\sigma * M_{\zeta} \mathcal{U}_{t} \Phi\left(z_{1}, z_{2}\right)\right|^{2} \\
& \left.\times\left(1+\left|z_{1}+(1-t) \zeta_{2}\right|+\left|z_{2}-t \zeta_{1}\right|\right)^{2 s} d z d \zeta\right)^{1 / 2} .
\end{aligned}
$$

This implies the desired result for the weight (1.4), since the last quantity is equivalent to the norm of $\sigma$ in $M_{m}^{2,2}$ (see Definition 1.1 and the paragraph just below it).

In the same way as above, the estimate obtained in Part II yields the result for the weight (1.5) for all $t \in \mathbf{R}$.

To prove Theorem 1.4, we use the following lemma.

Lemma $3.2\left(\left[5\right.\right.$, Lemma 2.3]). Let $\varphi_{0}(z)=e^{-\pi z^{2}}, z \in \mathbf{R}^{n}$. Then for all $p>0, r>0$ and $\left(z_{0}, \zeta_{0}\right) \in \mathbf{R}^{n} \times \mathbf{R}^{n}$, we have

$$
\left|f * M_{\zeta_{0}} \varphi_{0}\left(z_{0}\right)\right|^{p} \leq \frac{e^{p \pi r^{2} / 2}}{|B(r)|} \int_{\left(z-z_{0}\right)^{2}+\left(\zeta-\zeta_{0}\right)^{2}<r^{2}}\left|f * M_{\zeta} \varphi_{0}(z)\right|^{p} d z d \zeta
$$

where $|B(r)|$ is the volume of the ball with radius $r$ in $\mathbf{R}^{2 n}$.

Proof of Theorem 1.4. We first prove the result for the case $t=0$. Let $\left\{g_{m n}\right\}$ be the Gabor frame as given in Example 2.2. By the same reason as in the proof of Theorem 1.2, it is sufficient to prove the inequality

$$
\left(\sum_{m, n \in \mathbf{Z}^{d}}\left\|\sigma(X, D) g_{m n}\right\|_{L^{2}}^{p}\right)^{1 / p} \leq C\|\sigma\|_{M^{p, p}}
$$

for $\sigma \in \mathcal{S}\left(\mathbf{R}^{2 d}\right)$.

Taking $\Phi, \Psi \in \mathcal{S}\left(\mathbf{R}^{2 d}\right)$ as in the proof of Theorem 1.2, we have the estimate (3.5) and thus

$$
\begin{aligned}
& \sum_{m, n \in \mathbf{Z}^{d}}\left\|\sigma(X, D) g_{m n}\right\|_{L^{2}}^{p} \\
& \quad \lesssim \sum_{m, n \in \mathbf{Z}^{d}}\left(\int_{\mathbf{R}^{4 d}}\left|\sigma * M_{\zeta} \Phi(z)\right|^{2}\left\langle z_{1}+\zeta_{2}-m\right\rangle^{-N}\left\langle z_{2}-\frac{n}{2}\right\rangle^{-N} d z d \zeta\right)^{p / 2} \\
& \quad=(*)
\end{aligned}
$$

To proceed further, we take the special choice of $\Phi$ as

$$
\Phi(z)=\Phi_{0}(z)=e^{-\pi z^{2}}, \quad z \in \mathbf{R}^{2 d}
$$


and estimate $(*)$ with $\Phi=\Phi_{0}$ as follows:

$$
\begin{aligned}
& (*) \lesssim \sum_{m, n \in \mathbf{Z}^{d}} \\
& \times\left(\sum_{k, l \in \mathbf{Z}^{2 d}} \sup _{\substack{z \in k+[0,1]^{2 d} \\
\zeta \in l+[0,1]^{2 d}}}\left|\sigma * M_{\zeta} \Phi_{0}(z)\right|^{2}\left\langle z_{1}+\zeta_{2}-m\right\rangle^{-N}\left\langle z_{2}-\frac{n}{2}\right\rangle^{-N}\right)^{p / 2} \\
& \approx \sum_{m, n \in \mathbf{Z}^{d}} \\
& \times\left(\sum_{k, l \in \mathbf{Z}^{2 d}} \sup _{\substack{z \in k+[0,1]^{2 d} \\
\zeta \in l+[0,1]^{2 d}}}\left|\sigma *\left(M_{\zeta} \Phi_{0}\right)(z)\right|^{2}\left\langle k_{1}+l_{2}-m\right\rangle^{-N}\left\langle k_{2}-\frac{n}{2}\right\rangle^{-N}\right)^{p / 2} \\
& \leq \sum_{m, n \in \mathbf{Z}^{d}} \\
& \times \sum_{k, l \in \mathbf{Z}^{2 d}} \sup _{\substack{z \in k+[0,1]^{2 d} \\
\zeta \in l+[0,1]^{2 d}}}\left|\sigma * M_{\zeta} \Phi_{0}(z)\right|^{p}\left\langle k_{1}+l_{2}-m\right\rangle^{-N p / 2}\left\langle k_{2}-\frac{n}{2}\right\rangle^{-N p / 2} \\
& \approx \sum_{k, l \in \mathbf{Z}^{2 d}} \sup _{\substack{z \in k+[0,1]^{2 d} \\
\zeta \in l+[0,1]^{2 d}}}\left|\sigma * M_{\zeta} \Phi_{0}(z)\right|^{p}
\end{aligned}
$$

where we used the assumption that $p \leq 2$ to obtain the $\leq$. By Lemma 3.2, the last sum is majorized by a constant times

$$
\begin{gathered}
\sum_{k, l \in \mathbf{Z}^{2 d}} \int_{(z-k)^{2}+(\zeta-l)^{2} \leq 16 d}\left|\sigma * M_{\zeta} \Phi_{0}(z)\right|^{p} d z d \zeta \\
\approx \int_{\mathbf{R}^{4 d}}\left|\sigma * M_{\zeta} \Phi_{0}(z)\right|^{p} d z d \zeta \approx\|\sigma\|_{M^{p, p}}^{p}
\end{gathered}
$$

This proves the result for $\sigma(X, D)$.

Next we prove the result for general $t \in \mathbf{R}$. We use the operator $\mathcal{U}_{t}$ used in the proof of Theorem 1.2. Since $\sigma_{t}(X, D)=\left(\mathcal{U}_{t} \sigma\right)(X, D)$, it is sufficient to prove that the operator $\mathcal{U}_{t}$ is bounded in $M^{p, p}\left(\mathbf{R}^{2 d}\right)$.

Taking a $\Phi \in \mathcal{S}\left(\mathbf{R}^{2 d}\right)$, we have (3.7) and hence

$$
\int_{\mathbf{R}^{4 d}}\left|\left(\mathcal{U}_{t} \sigma\right) * M_{\zeta} \Phi(z)\right|^{p} d z d \zeta=\int_{\mathbf{R}^{4 d}}\left|\sigma * M_{\zeta} \mathcal{U}_{t} \Phi(z)\right|^{p} d z d \zeta
$$


Then we have $\left\|\mathcal{U}_{t} \sigma\right\|_{M^{p, p}} \approx\|\sigma\|_{M^{p, p}}$, since $\mathcal{U}_{t} \Phi \in \mathcal{S}\left(\mathbf{R}^{2 d}\right) \backslash\{0\}$. This completes the proof of Theorem 1.4.

\section{§4. Proof of Theorem 1.3}

Proof. We will prove only (a) and (b), because we can treat (c) and (d) in the same way as (a) and (b). We take a $\Phi \in \mathcal{S}\left(\mathbf{R}^{2 d}\right) \backslash\{0\}$.

(a) Let $m(z, \zeta)=\left(1+\left|z_{1}+t \zeta_{2}\right|\right)^{s}, t \neq 0$. What we have to prove is

$$
\left\|\left\langle z_{1}+t \zeta_{2}\right\rangle^{s} \sigma * M_{\zeta} \Phi(z)\right\|_{L_{z, \zeta}^{2}\left(\mathbf{R}^{4 d}\right)} \approx\left\|\left(1+\left|\zeta_{2}\right|\right)^{s} F_{2} \tau_{1 / t}\left(y_{1}, \zeta_{2}\right)\right\|_{L_{y_{1}, \zeta_{2}}^{2}\left(\mathbf{R}^{2 d}\right)} .
$$

By complex interpolation with respect to the parameter $s$, it is sufficient to prove (4.1) for all nonnegative integers $s$. If $s$ is a nonnegative integer, then the right-hand side of (4.1) is equivalent to

$$
\sum_{|\alpha| \leq s}\left\|\partial_{y_{2}}^{\alpha}\left(\tau_{1 / t}\left(y_{1}, y_{2}\right)\right)\right\|_{L_{y_{1}, y_{2}}^{2}}
$$

Thus, we will prove that

$$
\left\|\left\langle z_{1}+t \zeta_{2}\right\rangle^{s} \sigma * M_{\zeta} \Phi(z)\right\|_{L_{z, \zeta}^{2}} \approx \sum_{|\alpha| \leq s}\left\|\partial_{y_{2}}^{\alpha}\left(\tau_{1 / t}\left(y_{1}, y_{2}\right)\right)\right\|_{L_{y_{1}, y_{2}}^{2}}
$$

for all nonnegative integers $s$.

We first prove that

$$
\left\|\left\langle z_{1}+t \zeta_{2}\right\rangle^{s} \sigma * M_{\zeta} \Phi(z)\right\|_{L_{z, \zeta}^{2}} \lesssim \sum_{|\alpha| \leq s}\left\|\partial_{y_{2}}^{\alpha}\left(\tau_{1 / t}\left(y_{1}, y_{2}\right)\right)\right\|_{L_{y_{1}, y_{2}}^{2}}
$$

Set $\Theta(y)=e^{-2 \pi i \frac{1}{t} y_{1} y_{2}} \Phi(y)$. Then,

$$
\begin{aligned}
\left|\sigma * M_{\zeta} \Phi(z)\right| & \\
= & \left|\int_{\mathbf{R}^{2 d}} \sigma(y) e^{2 \pi i \zeta(z-y)} \Phi(z-y) d y\right| \\
= & \mid \int_{\mathbf{R}^{2 d}} e^{-2 \pi i \frac{1}{t} y_{1} y_{2}} \tau_{1 / t}(y) e^{2 \pi i\left(\zeta_{1}\left(z_{1}-y_{1}\right)+\zeta_{2}\left(z_{2}-y_{2}\right)\right)} \\
= & \times e^{2 \pi i \frac{1}{t}\left(z_{1}-y_{1}\right)\left(z_{2}-y_{2}\right)} \Theta(z-y) d y \mid \\
& \int_{\mathbf{R}^{2 d}} \tau_{1 / t}(y) \Theta(z-y) e^{-2 \pi i\left(y_{1}\left(\frac{z_{2}}{t}+\zeta_{1}\right)+y_{2}\left(\frac{z_{1}}{t}+\zeta_{2}\right)\right)} d y \mid
\end{aligned}
$$


and thus, by integration by parts,

$$
\begin{gathered}
\left|(-2 \pi i)^{|\alpha|}\left(\frac{z_{1}}{t}+\zeta_{2}\right)^{\alpha} \sigma * M_{\zeta} \Phi(z)\right| \\
=\left|\int_{\mathbf{R}^{2 d}} \tau_{1 / t}(y) \Theta(z-y) \partial_{y_{2}}^{\alpha}\left[e^{-2 \pi i\left(y_{1}\left(\frac{z_{2}}{t}+\zeta_{1}\right)+y_{2}\left(\frac{z_{1}}{t}+\zeta_{2}\right)\right)}\right] d y\right| \\
=\left|\int_{\mathbf{R}^{2 d}} \partial_{y_{2}}^{\alpha}\left[\tau_{1 / t}(y) \Theta(z-y)\right] e^{-2 \pi i\left(y_{1}\left(\frac{z_{2}}{t}+\zeta_{1}\right)+y_{2}\left(\frac{z_{1}}{t}+\zeta_{2}\right)\right)} d y\right| \\
=\mid \int_{\mathbf{R}^{2 d}} \sum_{\alpha^{\prime}+\alpha^{\prime \prime}=\alpha}\left(\begin{array}{c}
\alpha \\
\alpha^{\prime}
\end{array}\right) \tau_{1 / t}^{\left(0, \alpha^{\prime}\right)}(y)(-1)^{|\alpha|^{\prime \prime}} \Theta^{\left(0, \alpha^{\prime \prime}\right)}(z-y) \\
\times e^{-2 \pi i\left(y_{1}\left(\frac{z_{2}}{t}+\zeta_{1}\right)+y_{2}\left(\frac{z_{1}}{t}+\zeta_{2}\right)\right)} d y \mid,
\end{gathered}
$$

where

$$
\tau_{1 / t}^{\left(0, \alpha^{\prime}\right)}(y)=\partial_{y_{2}}^{\alpha^{\prime}}\left(\tau_{1 / t}(y)\right) \quad \text { and } \quad \Theta^{\left(0, \alpha^{\prime \prime}\right)}(y)=\partial_{y_{2}}^{\alpha^{\prime \prime}}(\Theta(y))
$$

Taking the sum of (4.4) over $|\alpha| \leq s$, we obtain

$$
\begin{aligned}
\int_{\mathbf{R}^{4 d}}\left|\sigma * M_{\zeta} \Phi(z)\right|^{2}\left(1+\left|z_{1}+t \zeta_{2}\right|\right)^{2 s} d z d \zeta & \\
& \approx \sum_{|\alpha| \leq s} \int_{\mathbf{R}^{4 d}}\left|\left(\frac{z_{1}}{t}+\zeta_{2}\right)^{\alpha} \sigma * M_{\zeta} \Phi(z)\right|^{2} d z d \zeta \\
& \lesssim \sum_{\left|\alpha^{\prime}+\alpha^{\prime \prime}\right| \leq s} \int_{\mathbf{R}^{4 d}} \mid \int_{\mathbf{R}^{2 d}} \tau_{1 / t}^{\left(0, \alpha^{\prime}\right)}(y) \Theta^{\left(0, \alpha^{\prime \prime}\right)}(z-y) \\
& =\sum_{\left|\alpha^{\prime}+\alpha^{\prime \prime}\right| \leq s} \int_{\mathbf{R}^{4 d}}\left|\tau_{1 / t}^{\left(0, \alpha^{\prime}\right)} * M_{\left(\frac{z_{2}}{t}+\zeta_{1}, \frac{z_{1}}{t}+\zeta_{2}\right)} \Theta^{\left(0, \alpha^{\prime \prime}\right)}(z)\right|^{2} d z d \zeta \\
& =\sum_{\left|\alpha^{\prime}+\alpha^{\prime \prime}\right| \leq s} \int_{\mathbf{R}^{4 d}}\left|\tau_{1 / t}^{\left(0, \alpha^{\prime}\right)} * M_{\zeta} \Theta^{\left(0, \alpha^{\prime \prime}\right)}(z)\right|^{2} d z d \zeta \\
& \approx \sum_{|\alpha| \leq s}\left\|\tau_{1 / t}^{(0, \alpha)}\right\|_{M^{2,2}}^{2} \approx \sum_{|\alpha| \leq s}\left\|\partial_{y_{2}}^{\alpha}\left(\tau_{1 / t}\left(y_{1}, y_{2}\right)\right)\right\|_{L_{y_{1}, y_{2}}^{2}}^{2}
\end{aligned}
$$

where the last $\approx$ follows from the fact that $M^{2,2}=L^{2}$. This proves (4.3). 
Next, we prove the converse inequality

$$
\sum_{|\alpha| \leq s}\left\|\partial_{y_{2}}^{\alpha}\left(\tau_{1 / t}\left(y_{1}, y_{2}\right)\right)\right\|_{L_{y_{1}, y_{2}}^{2}} \lesssim\left\|\left\langle z_{1}+t \zeta_{2}\right\rangle^{s} \sigma * M_{\zeta} \Phi(z)\right\|_{L_{z, \zeta}^{2}}
$$

We prove this by induction on $s$.

When $s=0,(4.5)$ is obvious since

$$
\left\|\tau_{1 / t}\right\|_{L^{2}}=\|\sigma\|_{L^{2}} \approx\left\|\sigma * M_{\zeta} \Phi(z)\right\|_{L_{z, \zeta}^{2}}
$$

Assume that (4.5) holds for a nonnegative integer $s$. Let $\alpha$ be a multiindex with $|\alpha|=s+1$. From (4.4), we have

$$
\begin{aligned}
& \left|(-2 \pi i)^{|\alpha|}\left(\frac{z_{1}}{t}+\zeta_{2}\right)^{\alpha} \sigma * M_{\zeta} \Phi(z)\right| \\
& \geq\left|\int_{\mathbf{R}^{2 d}} \tau_{1 / t}^{(0, \alpha)}(y) \Theta(z-y) e^{-2 \pi i\left(y_{1}\left(\frac{z_{2}}{t}+\zeta_{1}\right)+y_{2}\left(\frac{z_{1}}{t}+\zeta_{2}\right)\right)} d y\right| \\
& \quad-\sum_{\substack{\alpha^{\prime}+\alpha^{\prime \prime}=\alpha \\
\alpha^{\prime \prime} \neq 0}}\left(\begin{array}{c}
\alpha \\
\alpha^{\prime}
\end{array}\right) \\
& \quad \times\left|\int_{\mathbf{R}^{2 d}} \tau_{1 / t}^{\left(0, \alpha^{\prime}\right)}(y) \Theta^{\left(0, \alpha^{\prime \prime}\right)}(z-y) e^{-2 \pi i\left(y_{1}\left(\frac{z_{2}}{t}+\zeta_{1}\right)+y_{2}\left(\frac{z_{1}}{t}+\zeta_{2}\right)\right)} d y\right| \\
& =\left|\tau_{1 / t}^{(0, \alpha)} * M_{\left(\frac{z_{2}}{t}+\zeta_{1}, \frac{z_{1}}{t}+\zeta_{2}\right)} \Theta(z)\right| \\
& \quad \sum_{\substack{\alpha^{\prime}+\alpha^{\prime \prime}=\alpha \\
\alpha^{\prime \prime} \neq 0}}\left(\begin{array}{c}
\alpha \\
\alpha^{\prime}
\end{array}\right)\left|\tau_{1 / t}^{\left(0, \alpha^{\prime}\right)} * M_{\left(\frac{z_{2}}{t}+\zeta_{1}, \frac{z_{1}}{t}+\zeta_{2}\right)} \Theta^{\left(0, \alpha^{\prime \prime}\right)}(z)\right| .
\end{aligned}
$$

Thus, taking the $L_{z, \zeta}^{2}$-norm and using the fact that $M^{2,2}=L^{2}$, we obtain

$$
\left\|(-2 \pi i)^{|\alpha|}\left(\frac{z_{1}}{t}+\zeta_{2}\right)^{\alpha} \sigma * M_{\zeta} \Phi(z)\right\|_{L_{z, \zeta}^{2}}+\sum_{\left|\alpha^{\prime}\right|<|\alpha|}\left\|\tau_{1 / t}^{\left(0, \alpha^{\prime}\right)}\right\|_{L^{2}} \gtrsim\left\|\tau_{1 / t}^{(0, \alpha)}\right\|_{L^{2}} .
$$

Since, obviously,

$$
\left\|\left\langle\frac{z_{1}}{t}+\zeta_{2}\right\rangle^{s+1} \sigma * M_{\zeta} \Phi(z)\right\|_{L_{z, \zeta}^{2}} \gtrsim\left\|(-2 \pi i)^{|\alpha|}\left(\frac{z_{1}}{t}+\zeta_{2}\right)^{\alpha} \sigma * M_{\zeta} \Phi(z)\right\|_{L_{z, \zeta}^{2}},
$$

and since the induction hypothesis implies that

$$
\left\|\left\langle\frac{z_{1}}{t}+\zeta_{2}\right\rangle^{s} \sigma * M_{\zeta} \Phi(z)\right\|_{L_{z, \zeta}^{2}} \gtrsim \sum_{\left|\alpha^{\prime}\right|<|\alpha|}\left\|\tau_{1 / t}^{\left(0, \alpha^{\prime}\right)}\right\|_{L^{2}}
$$


we obtain

$$
\left\|\left\langle\frac{z_{1}}{t}+\zeta_{2}\right\rangle^{s+1} \sigma * M_{\zeta} \Phi(z)\right\|_{L_{z, \zeta}^{2}} \gtrsim\left\|\tau_{1 / t}^{(0, \alpha)}\right\|_{L^{2}}
$$

Thus, we proved (4.5).

(b) Let $m(z, \zeta)=\left(1+\left|z_{1}\right|\right)^{s}, s \geq 0$. We have

$$
\left|\sigma * M_{\zeta} \Phi(z)\right|=\left|\int_{\mathbf{R}^{2 d}} \sigma(y) e^{2 \pi i \zeta(z-y)} \Phi(z-y) d y\right|=\left|(\sigma(\cdot) \Phi(z-\cdot))^{\wedge}(\zeta)\right| .
$$

Hence, by Plancherel's theorem,

$$
\int_{\mathbf{R}^{2 d}}\left|\sigma * M_{\zeta} \Phi(z)\right|^{2} d \zeta=\int_{\mathbf{R}^{2 d}}|\sigma(y) \Phi(z-y)|^{2} d y,
$$

and consequently,

$$
\begin{aligned}
\|\sigma\|_{M_{m}^{2,2}}^{2} & =\int_{\mathbf{R}^{4 d}}\left|\sigma * M_{\zeta} \Phi(z)\right|^{2}\left(1+\left|z_{1}\right|\right)^{2 s} d \zeta d z \\
& =\int_{\mathbf{R}^{4 d}}|\sigma(y) \Phi(z-y)|^{2}\left(1+\left|z_{1}\right|\right)^{2 s} d y d z=(I) .
\end{aligned}
$$

By the inequality

$$
\left(1+\left|z_{1}-y_{1}\right|\right)^{-2 s} \leq \frac{\left(1+\left|z_{1}\right|\right)^{2 s}}{\left(1+\left|y_{1}\right|\right)^{2 s}} \leq\left(1+\left|z_{1}-y_{1}\right|\right)^{2 s},
$$

we obtain

$$
\begin{aligned}
(I) & \leq \int_{\mathbf{R}^{4 d}}|\sigma(y) \Phi(z-y)|^{2}\left(1+\left|z_{1}-y_{1}\right|\right)^{2 s}\left(1+\left|y_{1}\right|\right)^{2 s} d y d z \\
& \approx \int_{\mathbf{R}^{2 d}}|\sigma(y)|^{2}\left(1+\left|y_{1}\right|\right)^{2 s} d y
\end{aligned}
$$

and

$$
\begin{aligned}
(I) & \geq \int_{\mathbf{R}^{4 d}}|\sigma(y) \Phi(z-y)|^{2}\left(1+\left|z_{1}-y_{1}\right|\right)^{-2 s}\left(1+\left|y_{1}\right|\right)^{2 s} d y d z \\
& \approx \int_{\mathbf{R}^{2 d}}|\sigma(y)|^{2}\left(1+\left|y_{1}\right|\right)^{2 s} d y .
\end{aligned}
$$

Combining the above inequalities, we obtain

$$
\|\sigma\|_{M_{s}^{2,2}}^{2} \approx \int_{\mathbf{R}^{2 d}}|\sigma(y)|^{2}\left(1+\left|y_{1}\right|\right)^{2 s} d y
$$




\section{$\S 5$. Alternate proofs of Theorems 1.2 and 1.4}

An alternate proof of Theorem 1.2. We will see that Theorem 1.2 can be proved by a modification of the argument of Heil, Ramanathan, and Topiwala [12] or Gröchenig and Heil [9]. We follow [12] and give the proof of Theorem 1.2 for the case $t=1 / 2$; the case of general $t \in \mathbf{R}$ can be reduced to the case $t=1 / 2$ with the aid of the operator $\mathcal{U}_{t}$ as in Part III of the proof of Theorem 1.2 (Section 3). Since the essential argument is the same as [12, Sections 2-5], we omit some details. We use the notation

$$
\sigma^{W}(X, D)=\sigma_{1 / 2}(X, D)
$$

The argument of [12] (and [9] as well) is based on the fact that the inequality

$$
\sum_{j>\operatorname{rank}(T)} s_{j}(A)^{2} \leq\|A-T\|_{C_{2}}^{2}
$$

holds for all compact operators $A$ and for all finite-rank operators $T$. Thus, given a compact operator $A$, if we find a finite-rank operator $T$ which approximates $A$ well, then we can obtain an estimate of the singular values of $A$. To find a finite-rank approximation of $A=\sigma^{W}(X, D)$, we use a Gabor frame expansion of the symbol $\sigma$.

To begin with, we recall some basic facts on the Weyl correspondence (for details, see, e.g., [4]). For $\alpha=\left(\alpha_{1}, \alpha_{2}\right) \in \mathbf{R}^{2 d}$, we define the unitary operator $\rho(\alpha)$ in $L^{2}\left(\mathbf{R}^{d}\right)$ by

$$
\rho(\alpha) f(x)=e^{\pi i \alpha_{1} \alpha_{2}} e^{2 \pi i \alpha_{2} x} f\left(x+\alpha_{1}\right) .
$$

For $\varphi, \psi \in L^{2}\left(\mathbf{R}^{d}\right)$, the function $W(\varphi, \psi)$ on $\mathbf{R}^{2 d}$ is defined by

$$
W(\varphi, \psi)(x, \omega)=\int_{\mathbf{R}^{d}} e^{-2 \pi i p \omega} \varphi(x+p / 2) \overline{\psi(x-p / 2)} d p .
$$

This function is called the Wigner distribution. (Notice that we are writing the variables $x$ and $\omega$ in this order; in [4] and [12], the Wigner distribution is written as $W(\varphi, \psi)(\omega, x)$.) We have the formula

$$
\begin{aligned}
& W(\rho(\alpha) \varphi, \rho(\beta) \psi)(x, \omega) \\
& \quad=e^{\pi i\left(-\alpha_{2} \beta_{1}+\alpha_{1} \beta_{2}\right)} e^{2 \pi i\left\{\zeta_{1}\left(x+z_{1}\right)+\zeta_{2}\left(\omega+z_{2}\right)\right\}} W(\varphi, \psi)\left(x+z_{1}, \omega+z_{2}\right),
\end{aligned}
$$


where

$$
z_{1}=\frac{\alpha_{1}+\beta_{1}}{2}, \quad z_{2}=-\frac{\alpha_{2}+\beta_{2}}{2}, \quad \zeta_{1}=\alpha_{2}-\beta_{2}, \quad \zeta_{2}=\alpha_{1}-\beta_{1} .
$$

Using the notation of translation and modulation on $\mathbf{R}^{2 d}$, the above formula can be written as

$$
W(\rho(\alpha) \varphi, \rho(\beta) \psi)=e^{\pi i\left(-\alpha_{2} \beta_{1}+\alpha_{1} \beta_{2}\right)} T_{-z} M_{\zeta} W(\varphi, \psi) .
$$

We write $A$ to denote the linear mapping $\mathbf{R}^{4 d} \rightarrow \mathbf{R}^{4 d}$ defined by

$$
A:\left(\alpha_{1}, \alpha_{2}, \beta_{1}, \beta_{2}\right) \mapsto\left(z_{1}, z_{2}, \zeta_{1}, \zeta_{2}\right) \quad \text { with (5.2). }
$$

For our purpose, the Wigner distribution is useful since the pseudodifferential operator corresponding to $W(\varphi, \psi)$ is given by the following simple formula:

$$
W(\varphi, \psi)^{W}(X, D) f=\langle f, \psi\rangle \varphi .
$$

We also need the formula

$$
\left\|\sigma^{W}(X, D)\right\|_{C_{2}}=\|\sigma\|_{L^{2}} .
$$

Next, we recall some facts from the frame theory (for details, see [7], [11]). We take a function $\phi \in \mathcal{S}\left(\mathbf{R}^{d}\right)$ and a discrete subgroup $\Lambda$ of $\mathbf{R}^{2 d}$ such that the functions

$$
\phi_{\alpha}=\rho(\alpha) \phi, \quad \alpha \in \Lambda,
$$

form a frame for $L^{2}\left(\mathbf{R}^{d}\right)$. We set

$$
\begin{aligned}
\Phi_{\alpha, \beta} & =W\left(\phi_{\alpha}, \phi_{\beta}\right), \quad(\alpha, \beta) \in \Gamma=\Lambda \times \Lambda, \\
\Phi & =W(\phi, \phi) .
\end{aligned}
$$

By (5.3), we have

$$
\Phi_{\alpha, \beta}=e^{\pi i\left(-\alpha_{2} \beta_{1}+\alpha_{1} \beta_{2}\right)} T_{-z} M_{\zeta} \Phi, \quad(z, \zeta)=A(\alpha, \beta) .
$$

The following are known in the theory of frames. The set $\left\{\Phi_{\alpha, \beta}\right\}_{(\alpha, \beta) \in \Gamma}$ is a frame for $L^{2}\left(\mathbf{R}^{2 d}\right)$. The dual frame of $\left\{\phi_{\alpha}\right\}_{\alpha \in \Lambda}$ is also of the form $\{\rho(\alpha) \widetilde{\phi}\}_{\alpha \in \Lambda}$ with a $\widetilde{\phi} \in \mathcal{S}\left(\mathbf{R}^{d}\right)$; we write $\widetilde{\phi}_{\alpha}=\rho(\alpha) \widetilde{\phi}$. The dual frame of $\left\{\Phi_{\alpha, \beta}\right\}_{(\alpha, \beta) \in \Gamma}$ is given by

$$
\widetilde{\Phi}_{\alpha, \beta}=W\left(\widetilde{\phi}_{\alpha}, \widetilde{\phi}_{\beta}\right), \quad(\alpha, \beta) \in \Gamma .
$$


Every $\sigma \in L^{2}\left(\mathbf{R}^{2 d}\right)$ can be decomposed as

$$
\sigma=\sum_{(\alpha, \beta) \in \Gamma}\left\langle\sigma, \Phi_{\alpha, \beta}\right\rangle \widetilde{\Phi}_{\alpha, \beta}
$$

with the series converging unconditionally in $L^{2}\left(\mathbf{R}^{2 d}\right)$.

Now, in order to get a finite-rank approximation of $\sigma^{W}(X, D)$, we take a subset $\Gamma_{N} \subset \Gamma, N \in \mathbf{N}$, and define

$$
\sigma_{N}=\sum_{(\alpha, \beta) \in \Gamma_{N}}\left\langle\sigma, \Phi_{\alpha, \beta}\right\rangle \widetilde{\Phi}_{\alpha, \beta} .
$$

By (5.4) and (5.7), the operator $\sigma_{N}^{W}(X, D)$ is given by

$$
\sigma_{N}^{W}(X, D): f \mapsto \sum_{(\alpha, \beta) \in \Gamma_{N}}\left\langle\sigma, \Phi_{\alpha, \beta}\right\rangle\left\langle f, \widetilde{\phi}_{\beta}\right\rangle \widetilde{\phi}_{\alpha}
$$

Thus, if $\left\{\alpha \mid(\alpha, \beta) \in \Gamma_{N}\right\}$ or $\left\{\beta \mid(\alpha, \beta) \in \Gamma_{N}\right\}$ is a finite set, then $\sigma_{N}^{W}(X, D)$ is a finite-rank operator, and we have

$$
\operatorname{rank}\left(\sigma_{N}^{W}(X, D)\right) \leq \min \left\{\sharp\left\{\alpha \mid(\alpha, \beta) \in \Gamma_{N}\right\}, \sharp\left\{\beta \mid(\alpha, \beta) \in \Gamma_{N}\right\}\right\},
$$

where $\sharp E$ denotes the cardinality of a set $E$. By (5.5) and by the expansions (5.8) and (5.9), we have

$$
\begin{aligned}
\left\|\sigma^{W}(X, D)-\sigma_{N}^{W}(X, D)\right\|_{C_{2}}^{2} & =\left\|\sigma-\sigma_{N}\right\|_{L^{2}\left(\mathbf{R}^{2 d}\right)}^{2} \\
& =\left\|\sum_{(\alpha, \beta) \in \Gamma \backslash \Gamma_{N}}\left\langle\sigma, \Phi_{\alpha, \beta}\right\rangle \widetilde{\Phi}_{\alpha, \beta}\right\|_{L^{2}\left(\mathbf{R}^{2 d}\right)}^{2} \\
& \leq c \sum_{(\alpha, \beta) \in \Gamma \backslash \Gamma_{N}}\left|\left\langle\sigma, \Phi_{\alpha, \beta}\right\rangle\right|^{2},
\end{aligned}
$$

where the last inequality follows from the fact that $\left\{\widetilde{\Phi}_{\alpha, \beta}\right\}$ is a frame for $L^{2}\left(\mathbf{R}^{2 d}\right)$. We simply write

$$
\gamma(z, \zeta)=\left\langle\sigma, T_{z} M_{\zeta} \Phi\right\rangle
$$

By (5.6),

$$
\left|\left\langle\sigma, \Phi_{\alpha, \beta}\right\rangle\right|=\left|\left\langle\sigma, T_{-z} M_{\zeta} \Phi\right\rangle\right|=|\gamma(-z, \zeta)|, \quad(z, \zeta)=A(\alpha, \beta),
$$


and the above inequality can be written as

$$
\left\|\sigma^{W}(X, D)-\sigma_{N}^{W}(X, D)\right\|_{C_{2}}^{2} \leq c \sum_{(z, \zeta) \in A(\Gamma) \backslash A\left(\Gamma_{N}\right)}|\gamma(-z, \zeta)|^{2} .
$$

Applying the general principle (5.1), we obtain

$$
\sum_{j>k_{N}} s_{j}\left(\sigma^{W}(X, D)\right)^{2} \leq c \sum_{(z, \zeta) \in A(\Gamma) \backslash A\left(\Gamma_{N}\right)}|\gamma(-z, \zeta)|^{2},
$$

where $k_{N}=\operatorname{rank}\left(\sigma_{N}^{W}(X, D)\right)$.

To get an estimate of $k_{N}$, we take

$$
\Gamma_{N}=\left\{(\alpha, \beta) \in \Gamma|| \alpha_{1}|+| \alpha_{2} \mid \leq N\right\} .
$$

Then, by (5.10),

$$
k_{N} \leq \sharp\left\{\alpha \mid(\alpha, \beta) \in \Gamma_{N}\right\} \leq c_{0} N^{2 d},
$$

where $c_{0}$ is a constant depending only on $d$. From (5.2), we have

$$
A\left(\Gamma_{N}\right)=\left\{(z, \zeta) \in A(\Gamma)|| z_{1}+\zeta_{2} / 2|+|-z_{2}+\zeta_{1} / 2 \mid \leq N\right\} .
$$

Thus,

$$
\begin{aligned}
& \sum_{(z, \zeta) \in A(\Gamma) \backslash A\left(\Gamma_{N}\right)}|\gamma(-z, \zeta)|^{2} \\
& \leq N^{-2 s} \sum_{(z, \zeta) \in A(\Gamma) \backslash A\left(\Gamma_{N}\right)}|\gamma(-z, \zeta)|^{2}\left(1+\left|z_{1}+\zeta_{2} / 2\right|+\left|-z_{2}+\zeta_{1} / 2\right|\right)^{2 s} \\
& =N^{-2 s} \sum_{(z, \zeta) \in A(\Gamma) \backslash A\left(\Gamma_{N}\right)}|\gamma(-z, \zeta)|^{2} m(-z, \zeta)^{2},
\end{aligned}
$$

where $m$ is the weight function of (1.5) with $t=1 / 2$. Combining the above inequalities, we obtain

$$
\sum_{j>c_{0} N^{2 d}} s_{j}\left(\sigma^{W}(X, D)\right)^{2} \leq c N^{-2 s} \sum_{(z, \zeta) \in A(\Gamma) \backslash A\left(\Gamma_{N}\right)}|\gamma(-z, \zeta)|^{2} m(-z, \zeta)^{2} .
$$

From this inequality and from the fact that $\left\{s_{j}(A)\right\}$ is a nonincreasing sequence, we obtain the estimate

$$
s_{k}\left(\sigma^{W}(X, D)\right) \leq c k^{-(s+d) / 2 d}\left(\sum_{(z, \zeta) \in A(\Gamma)}|\gamma(-z, \zeta)|^{2} m(-z, \zeta)^{2}\right)^{1 / 2} .
$$


Thus, if we have the inequality

$$
\left(\sum_{(z, \zeta) \in A(\Gamma)}|\gamma(-z, \zeta)|^{2} m(-z, \zeta)^{2}\right)^{1 / 2} \leq c\|\sigma\|_{M_{m}^{2,2}}
$$

then we have

$$
s_{k}\left(\sigma^{W}(X, D)\right) \leq c k^{-(s+d) / 2 d}\|\sigma\|_{M_{m}^{2,2}} .
$$

If in addition $(s+d) / 2 d>p$, then we have

$$
\left\|\sigma^{W}(X, D)\right\|_{C_{p}} \leq c\left\|\left\{k^{-(s+d) / 2 d}\right\}\right\|_{l^{p}}\|\sigma\|_{M_{m}^{2,2}}=c\|\sigma\|_{M_{m}^{2,2}}
$$

which implies the claim of Theorem 1.2 for $t=1 / 2$ with the weight (1.5).

If we take

$$
\Gamma_{N}=\left\{(\alpha, \beta) \in \Gamma|| \beta_{1}|+| \beta_{2} \mid \leq N\right\},
$$

then we also have $k_{N}=\operatorname{rank}\left(\sigma_{N}^{W}(X, D)\right) \leq c_{0} N^{2 d}$ and

$$
A\left(\Gamma_{N}\right)=\left\{(z, \zeta) \in A(\Gamma)|| z_{1}-\zeta_{2} / 2|+|-z_{2}-\zeta_{1} / 2 \mid \leq N\right\} .
$$

Hence, by the same argument as above, we obtain

$$
\begin{aligned}
& s_{k}\left(\sigma^{W}(X, D)\right) \\
& \leq c k^{-(s+d) / 2 d} \\
& \times\left(\sum_{(z, \zeta) \in A(\Gamma)}|\gamma(-z, \zeta)|^{2}\left(1+\left|z_{1}-\zeta_{2} / 2\right|+\left|-z_{2}-\zeta_{1} / 2\right|\right)^{2 s}\right)^{1 / 2} \\
&= c k^{-(s+d) / 2 d}\left(\sum_{(z, \zeta) \in A(\Gamma)}|\gamma(-z, \zeta)|^{2} m(-z, \zeta)^{2}\right)^{1 / 2}
\end{aligned}
$$

where $m$ is the weight function of (1.4) with $t=1 / 2$. Hence, if inequality (5.11) holds, then the claim of Theorem 1.2 for $t=1 / 2$ with weight (1.4) follows.

Thus, the rest of the proof is to show the inequality (5.11) for the weight functions of (1.4) and (1.5). This can be done at least for the following special choice of $\phi$ and $\Lambda$ :

$$
\begin{aligned}
\phi(x) & =2^{d / 4} e^{-\pi x^{2}} \quad\left(x \in \mathbf{R}^{d}\right), \\
\Lambda & =a \mathbf{Z}^{d} \times b \mathbf{Z}^{d}, \quad a, b>0, a b<1 .
\end{aligned}
$$


In fact, for the above $\phi$ and $\Lambda$, it is known that $\{\rho(\alpha) \phi\}_{\alpha \in \Lambda}$ is a frame for $L^{2}\left(\mathbf{R}^{d}\right)$; this fact is due to Seip and Wallstén ([21], [22]). We have

$$
\Phi(x, \omega)=W(\phi, \phi)(x, \omega)=2^{d} e^{-2 \pi\left(x^{2}+\omega^{2}\right)},
$$

and for this $\Phi$, using the inequality of Lemma 3.2 and arguing in a similar way as in the proof of Theorem 1.4, we can prove inequality (5.11). This completes the proof.

Finally, we give a sketch of an alternate proof of Theorem 1.4 for the case $0<p \leq 1$.

An alternate proof of Theorem 1.4 for $0<p \leq 1$. With an appropriate pair $\Phi, \Psi \in \mathcal{S}\left(\mathbf{R}^{2 d}\right)$ and with appropriate $\alpha, \beta \in(0, \infty)$, we have the representation

$$
\sigma=\sum_{k, l \in \mathbf{Z}^{2 d}}\left\langle\sigma, T_{k \alpha} M_{l \beta} \Phi\right\rangle T_{k \alpha} M_{l \beta} \Psi
$$

with

$$
\|\sigma\|_{M^{p, p}} \approx\left(\sum_{k, l \in \mathbf{Z}^{2 d}}\left|\left\langle\sigma, T_{k \alpha} M_{l \beta} \Phi\right\rangle\right|^{p}\right)^{1 / p}
$$

(see [5, Theorem 3.7]). We take $\varphi_{1}, \varphi_{2} \in \mathcal{S}\left(\mathbf{R}^{d}\right)$ and take $\Psi$ to be the Rihaczek distribution

$$
\Psi(x, \omega)=R\left(\varphi_{1}, \varphi_{2}\right)(x, \omega)=\varphi_{1}(x) \overline{\widehat{\varphi_{2}}(\omega)} e^{-2 \pi i x \omega}, \quad x, \omega \in \mathbf{R}^{d} .
$$

Then $T_{z} M_{\zeta} \Psi(X, D)$ has the following simple form:

$$
T_{z} M_{\zeta} \Psi(X, D) f(x)=e^{-2 \pi i \zeta_{1} z_{1}}\left\langle f, M_{z_{2}} T_{z_{1}+\zeta_{2}} \varphi_{2}\right\rangle M_{z_{2}+\zeta_{1}} T_{z_{1}} \varphi_{1}(x)
$$

(see [8, Lemma 8.35]). In particular, $T_{z} M_{\zeta} \Psi(X, D)$ is an operator of rank 1 , and

$$
\left\|T_{z} M_{\zeta} \Psi(X, D)\right\|_{C_{p}} \leq c
$$

with $c$ independent of $z$ and $\zeta$. We use the fact that $\|\cdot\|_{C_{p}}^{p}$ is subadditive for $0<p \leq 1$ :

$$
\|T+S\|_{C_{p}}^{p} \leq\|T\|_{C_{p}}^{p}+\|S\|_{C_{p}}^{p}, \quad 0<p \leq 1
$$


(this fact is due to McCarthy [15, Theorem 2.8]). Now combining the above results, we obtain, for $0<p \leq 1$,

$$
\begin{aligned}
\|\sigma(X, D)\|_{C_{p}}^{p} & =\left\|\sum_{k, l \in \mathbf{Z}^{2 d}}\left\langle\sigma, T_{k \alpha} M_{l \beta} \Phi\right\rangle T_{k \alpha} M_{l \beta} \Psi(X, D)\right\|_{C_{p}}^{p} \\
& \leq \sum_{k, l \in \mathbf{Z}^{2 d}}\left|\left\langle\sigma, T_{k \alpha} M_{l \beta} \Phi\right\rangle\right|^{p}\left\|T_{k \alpha} M_{l \beta} \Psi(X, D)\right\|_{C_{p}}^{p} \\
& \lesssim \sum_{k, l \in \mathbf{Z}^{2 d}}\left|\left\langle\sigma, T_{k \alpha} M_{l \beta} \Phi\right\rangle\right|^{p} \approx\|\sigma\|_{M^{p, p}}^{p} .
\end{aligned}
$$

In the above proof, the use of the Rihaczek distribution makes the argument simple but is not essential since, as the argument in the proof of Theorem 1.2 shows, the estimate (5.12) holds for arbitrary $\Psi \in \mathcal{S}\left(\mathbf{R}^{2 d}\right)$.

If we use Lemma 2.3, we can also avoid using the subadditivity (5.13). In fact, the argument in the proof of Theorem 1.2 shows that, for the frame $\left\{g_{m n}\right\}$ of Example 2.2,

$$
\left\|T_{z} M_{\zeta} \Psi(X, D) g_{m n}\right\|_{L^{2}}^{2} \leq c\left\langle z_{1}+\zeta_{2}-m\right\rangle^{-N}\left\langle z_{2}-n / 2\right\rangle^{-N}
$$

(see (3.5)), which implies that

$$
\sum_{m, n \in \mathbf{Z}^{d}}\left\|T_{z} M_{\zeta} \Psi(X, D) g_{m n}\right\|_{L^{2}}^{p} \leq c .
$$

Hence, if $0<p \leq 1$, we have

$$
\begin{aligned}
\|\sigma(X, D)\|_{C^{p}}^{p} & \lesssim \sum_{m, n \in \mathbf{Z}^{d}}\left\|\sigma(X, D) g_{m n}\right\|_{L^{2}}^{p} \\
& \leq \sum_{m, n \in \mathbf{Z}^{d}}\left(\sum_{k, l \in \mathbf{Z}^{d}}\left|\left\langle\sigma, T_{k \alpha} M_{l \beta} \Phi\right\rangle\right|\left\|T_{k \alpha} M_{l \beta} \Psi(X, D) g_{m n}\right\|_{L^{2}}\right)^{p} \\
& \leq \sum_{m, n \in \mathbf{Z}^{d}} \sum_{k, l \in \mathbf{Z}^{d}}\left|\left\langle\sigma, T_{k \alpha} M_{l \beta} \Phi\right\rangle\right|^{p}\left\|T_{k \alpha} M_{l \beta} \Psi(X, D) g_{m n}\right\|_{L^{2}}^{p} \\
& \lesssim \sum_{k, l \in \mathbf{Z}^{d}}\left|\left\langle\sigma, T_{k \alpha} M_{l \beta} \Phi\right\rangle\right|^{p} \approx\|\sigma\|_{M^{p, p}}^{p}
\end{aligned}
$$

where the assumption that $p \leq 1$ is used to obtain the last $\leq$. 


\section{REFERENCES}

[1] G. Arsu, On Schatten-von Neumann class properties of pseudodifferential operators: The Cordes-Kato method, J. Operator Theory 59 (2008), 81-114.

[2] N. Dunford and J. T. Schwartz, Linear Operators, II: Spectral Theory, John Wiley, New York, 1963.

[3] H. G. Feichtinger, "Modulation spaces on locally compact abelian groups" in Wavelets and Their Applications, Allied, New Delhi, 2003, 99-140.

[4] G. B. Folland, Harmonic Analysis in Phase Space, Ann. of Math. Stud. 122, Princeton University Press, Princeton, 1989.

[5] Y. Galperin and S. Samarah, Time-frequency analysis on modulation spaces $M_{m}^{p, q}$, $0<p, q \leq \infty$, Appl. Comput. Harmon. Anal. 16 (2004), 1-18.

[6] K. Gröchenig, An uncertainty principle related to the Poisson summation formula, Studia Math. 121 (1996), 87-104.

[7] - Foundations of Time-Frequency Analysis, Birkhäuser, Boston, 2001.

[8] - "A pedestrian's approach to pseudodifferential operators" in Harmonic Analysis and Applications, Birkhäuser, Boston, 2006, 139-169.

[9] K. Gröchenig and C. Heil, Modulation spaces and pseudodifferential operators, Integral Equations Operator Theory 34 (1999), 439-457.

[10] - Counterexamples for boundedness of pseudodifferential operators, Osaka J. Math. 41 (2004), 681-691.

[11] C. Heil, History and evolution of the density theorem for Gabor frames, J. Fourier Anal. Appl. 13 (2007), 113-166.

[12] C. Heil, J. Ramanathan, and P. Topiwala, Singular values of compact pseudodifferential operators, J. Funct. Anal. 150 (1997), 426-452.

[13] M. Kobayashi, Modulation spaces $M^{p, q}$ for $0<p, q \leq \infty$, J. Funct. Spaces Appl. 4 (2006), 329-341.

[14] H. Komatsu, Theory of Locally Convex Spaces, Lecture Notes, Department of Math., Univ. of Tokyo, Tokyo, 1974.

[15] C. A. McCarthy, $C_{p}$, Israel J. Math. 5 (1967), 249-271.

[16] R. Meise and D. Vogt, Introduction to Functional Analysis, Oxf. Grad. Texts Math. 2, Oxford University Press, New York, 1997.

[17] H. Rauhut, Coorbit space theory for quasi-Banach spaces, Studia Math. 180 (2007), 237-253.

[18] R. Rochberg and K. Tachizawa, "Pseudodifferential operators, Gabor frames, and local trigonometric bases" in Gabor Analysis and Algorithms, Appl. Numer. Harmon. Anal., Birkhäuser, Boston, 1998, 171-192.

[19] C. Rondeaux, Classes de Schatten d'opérateurs pseudo-différentiels, Ann. Sci. Éc. Norm. Supér. (4) 17 (1984), 67-81.

[20] R. Schatten, Norm Ideals of Completely Continuous Operators, Ergeb. Math. Grenzgeb. (3) 27, Springer, Berlin, 1960.

[21] K. Seip, Density theorems for sampling and interpolation in the Bargman-Fock space, I, J. Reine. Angew. Math. 429 (1992), 91-106.

[22] K. Seip and R. Wallstén, Density theorems for sampling and interpolation in the Bargman-Fock space, II, J. Reine. Angew. Math. 429 (1992), 107-113. 
Masaharu Kobayashi

Department of Mathematics

Tokyo University of Science

Tokyo 162-8601

Japan

kobayashi@ma.kagu.tus.ac.jp

Akihiko Miyachi

Department of Mathematics

Tokyo Woman's Christian University

Tokyo 167-8585

Japan

miyachi@lab.twcu.ac.jp 